\title{
Convergence analysis of the FEM approximation of the first order projection method for incompressible flows with and without the inf-sup condition
}

\author{
Santiago Badia • Ramon Codina
}

Received: 5 September 2005 / Revised: 19 May 2006 / Published online: 11 July 2007

(C) Springer-Verlag 2007

\begin{abstract}
In this paper we obtain convergence results for the fully discrete projection method for the numerical approximation of the incompressible Navier-Stokes equations using a finite element approximation for the space discretization. We consider two situations. In the first one, the analysis relies on the satisfaction of the inf-sup condition for the velocity-pressure finite element spaces. After that, we study a fully discrete fractional step method using a Poisson equation for the pressure. In this case the velocity-pressure interpolations do not need to accomplish the inf-sup condition and in fact we consider the case in which equal velocity-pressure interpolation is used. Optimal convergence results in time and space have been obtained in both cases.
\end{abstract}

Mathematics Subject Classification (2000) $35 \mathrm{Q} 30 \cdot 65 \mathrm{M} 12 \cdot 65 \mathrm{M} 60$

\section{Introduction}

\subsection{State of the art}

The description of incompressible flow problems is determined by the Navier-Stokes equations. The numerical approach to this system of partial difference equations is a difficult task that has focused an active research in the last decades. The incompressibility constraint couples the velocity and pressure calculation, being its numerical solution expensive. Furthermore, the velocity and pressure interpolation spaces must

\footnotetext{
S. Badia $(\varangle) \cdot$ R. Codina

Universitat Politècnica de Catalunya, Jordi Girona 1-3, Edifici C1, 08034 Barcelona, Spain

e-mail:sbadia@cimne.upc.edu

R. Codina

e-mail: ramon.codina@upc.edu
} 
satisfy a compatibility condition for standard discretizations such as the Galerkin method.

This work is devoted to the analysis of the most classical pressure segregation method. Since its appearance in the late 1960's, with the pioneering works of Chorin [4] and Temam [26], this method has enjoyed a widespread popularity. Its key feature is the decoupling of the velocity and pressure calculation, yielding an important reduction of computational cost.

Another interesting issue of the classical projection method is its inherent pressure stability. It can be seen by numerical experimentation that equal order velocity and pressure space approximations lead to stable systems, so that the discrete inf-sup condition can be violated. A complete study of the machinery that makes the fully discretized projection method stable was developed in [6] and extended in [7]. The characterization of the stabilizer motivated the stabilization method proposed in $[8,9]$.

The fractional step method has been studied analytically in order to prove convergence and obtain error estimates. For periodic boundary conditions and a centered finite difference space approximation Chorin proved in [5] that the convergence was of first order in the time step size $\Delta t$, provided $\Delta t=\mathcal{O}\left(h^{2}\right), h$ being the diameter of the space discretization. A proof of convergence of the semi-discrete (space continuous) projection method to a continuous solution $\boldsymbol{u}(t)$ was given by Temam (see [26]) in a general setting.

The pioneering work of Shen [24] analyzed the semi-implicit version of the semidiscrete projection method, i.e. taking the convective term as $\boldsymbol{u} \cdot \nabla \hat{\boldsymbol{u}}^{n+1}$ (see the
notation introduced below). He obtained the first error estimates for the velocity,
but in a weak norm, $\boldsymbol{L}^{2}\left(0, T ; \boldsymbol{L}^{2}(\Omega)\right.$ ) (these spaces are defined later on). The proof
of the error estimates in this work is plagued by a mistake whep using dual porms
which was originally observed by Guermond [15]. In [25] these errors were corrected.

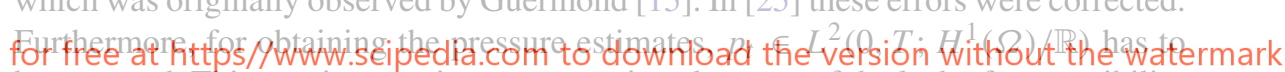
be assumed. This requirement is not appropriate, because of the lack of compatibility with the given data at time $t \rightarrow 0$ (see [27]).

Rannacher proposed an alternative version of the projection method in [23]. In this new format the end-of-step velocity disappears, and the problem can be understood as a penalized method with a lag in the evaluation of the pressure in the momentum equation. Based on this approach, Prohl obtained sharp error estimates for the semidiscrete in time projection method (see [22]). For instance, first order error estimates for the velocity were obtained with a stronger norm, $L^{\infty}\left(0, T ; L^{2}(\Omega)\right)$.

A modified scheme was proposed and analyzed by Shen [24]. It consists of introducing in the momentum equation an approximation of the pressure, more specifically, the term $\nabla p^{n}$. This method is usually called incremental projection method. As before, the pressure can be obtained from a Poisson equation. However, the value obtained in this case is a correction of the pressure $\delta p^{n+1}$ instead of the total pressure $p^{n+1}$. This scheme is also called pressure correction method. Shen obtained improved error estimates for this method in [24]. Both the intermediate $\hat{\boldsymbol{u}}^{n+1}$ and end-of-step $\boldsymbol{u}^{n+1}$ velocities are first order approximations to the continuous velocities in $L^{\infty}\left(0, T ; \boldsymbol{L}^{2}(\Omega)\right)$. Error estimates for a fully discrete incremental scheme were introduced in $[16,17]$. Therein, the pressure is not calculated from a Poisson equation, but from a Stokes problem. This analysis requires velocity and pressure interpolations satisfying the inf- 
sup condition for obtaining error estimates of first order in the time step size and of optimal order in the mesh size.

\subsection{Novel results, road map of the proofs and outline of the article}

Even though the semi-discretized (in time) version of the classical projection method has attracted a lot of research, its fully discretized version has not been fairly analyzed. The present work has been carried out with the aim to perform the very first analysis of this widely used method discretized in space using finite elements. Furthermore, at the fully discrete level we can address questions that do not have sense at the semi-discrete level. Probably the most salient is: is it possible to get optimal error estimates without relying on the inf-sup condition? In order to answer this question, we have obtained error estimates when using a pressure Poisson equation (the most appealing scheme from a computational point of view) without relying on the inf-sup condition. Another aspect of special interest is the analysis of the algebraic projection method (see [21]). A main difference of this method (split at the fully discrete level) with respect to the classical one is the fact that with this method the end-of-step velocity satisfies the full Dirichlet boundary conditions. We point out how this fact affects the analysis.

Let us sketch the main results of this paper, and the strategy pursued for the obtention of these results. We start introducing an auxiliary problem that consists of a

semi-discrete (in tine is evaluated exactly

convergence and stabili

jection method with t
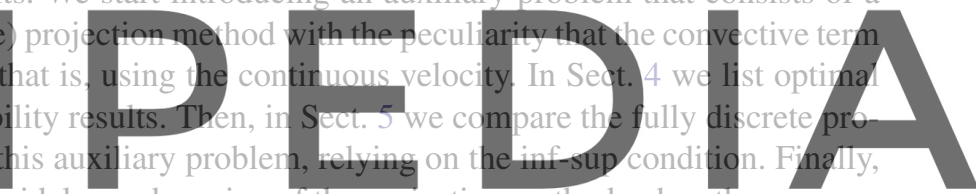

we analyze the most widely used version of the projection method, when the pressure

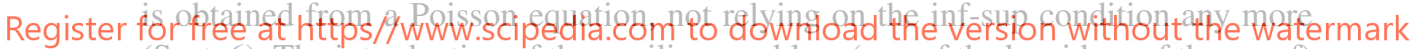
(Sect. 6). The introduction of the auxiliary problem (one of the key ideas of the proof) is justified when bounding the error related to the convective term, where $\mathbb{H}^{2}(\Omega)$ stability of the semi-discrete solution is required. In Table 1 we have summarized the main results in order to provide the reader with a road map for the subsequent analysis.

The paper is organized as follows: Assumptions for the continuous solution of the Navier-Stokes equations are stated and some regularity properties are presented in Sect. 2. The fractional step method to be analyzed is presented in Sect. 3. In Sect. 4 we introduce the auxiliary problem and some convergence and stability results are stated.

Table 1 List of main results

\begin{tabular}{lll}
\hline Method & Main result & Label \\
\hline Semi-discrete auxiliary problem & $\begin{array}{l}\text { Stability } \\
\text { Convergence }\end{array}$ & $\begin{array}{l}\text { Lemma 1 } \\
\text { Corollary 1 }\end{array}$ \\
$\begin{array}{l}\text { Fully discrete projection method } \\
\text { (with inf-sup) }\end{array}$ & Convergence & Corollary 2 \\
$\begin{array}{l}\text { Fully discrete projection method } \\
\text { (without inf-sup) }\end{array}$ & Convergence & Corollary 3 \\
\hline
\end{tabular}


Section 5 is devoted to the analysis of the fully discrete projection scheme satisfying the inf-sup condition. The fully discrete projection scheme using a Poisson equation for the pressure is presented in Sect. 6.

\section{Problem statement and preliminaries}

The evolution equations for an incompressible fluid moving in a domain $\Omega$ of $\mathbb{R}^{d}$ $(d=2$ or 3 ) in a time interval $[0, T]$ consists of finding a velocity $\boldsymbol{u}$ and a pressure $p$ such that

$$
\begin{aligned}
\partial_{t} \boldsymbol{u}-v \Delta \boldsymbol{u}+\boldsymbol{u} \cdot \nabla \boldsymbol{u}+\nabla p & =\boldsymbol{f} & & \text { in } \Omega \times(0, T), \\
\nabla \cdot \boldsymbol{u} & =0 & & \text { in } \Omega \times(0, T), \\
\boldsymbol{u} & =\mathbf{0} & & \text { on } \Gamma \times(0, T), \\
u(x, 0) & =u_{0} & & \text { in } \Omega \times\{0\} .
\end{aligned}
$$

where $f$ is the force vector, $v$ is the kinematic viscosity, $\boldsymbol{u}_{0}$ the initial condition and $\Gamma=\partial \Omega$. We will use $\partial_{t}$ or a subscript $t$ to denote the temporal partial derivative.

In order to obtain the weak form of problem (1) we need to introduce some notation. We denote by $L^{p}(\Omega)$ the $p$-th power absolu $L^{\infty}(\Omega)$ consists of interest; $L^{2}(\Omega)$ is induced norm $\|u\|_{0}$. Th
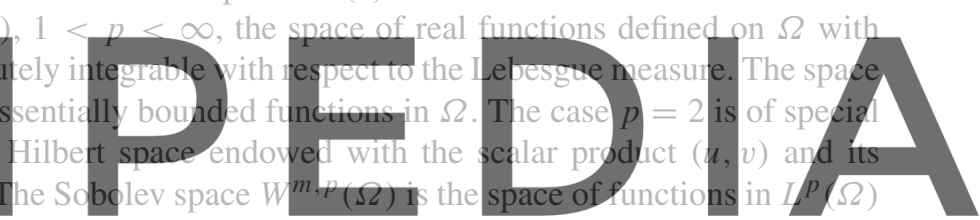

whose weak derivatives of order less than or equal to $m$ belong to $L^{p}(\Omega), m$ being

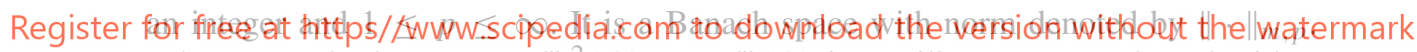

When $p=2$, the space $W^{m, 2}(\Omega)=H^{m}(\Omega)$ is a Hilbert space endowed with a scalar product and its associated nom $\|\cdot\| m$. Furthermore, we denote by $H_{0}^{1}(\Omega)$ the space of functions of $H^{1}(\Omega)$ vanishing on $\Gamma$ and by $H^{-1}(\Omega)$ its dual space. In general, duality pairings will be denoted with the symbol $\langle\cdot, \cdot\rangle$. We point out that the seminorn $|u|_{1}=\|\nabla u\|_{0}$ in $H^{1}(\Omega)$ is a norm in $H_{0}^{1}(\Omega)$. From now onwards we will not distinguish between $\|u\|_{1}$ and $\|\nabla u\|_{0}$ for $u \in H_{0}^{1}(\Omega)$.

We shall often consider $d$-dimensional vector functions with components in one of these spaces. We shall indicate them by boldface letters, for instance $\boldsymbol{H}^{m}(\Omega)=$ $\left(H^{m}(\Omega)\right)^{d}$. In the next, we will not distinguish between scalar products or norms for scalar or vector-valued functions.

Let us introduce some convenient spaces for the treatment of the incompressibility constraint. The first one is

$$
\boldsymbol{H}(\operatorname{div}, \Omega):=\left\{\boldsymbol{u} \in \boldsymbol{L}^{2}(\Omega) \mid \nabla \cdot \boldsymbol{u} \in L^{2}(\Omega)\right\}
$$

which is a Hilbert space with norm $\|\boldsymbol{u}\|_{\text {div }}=\|\boldsymbol{u}\|_{0}+\|\nabla \cdot \boldsymbol{u}\|_{0}$ and

$$
\boldsymbol{H}_{0}(\operatorname{div}, \Omega):=\left\{\boldsymbol{u} \in \boldsymbol{H}(\operatorname{div}, \Omega)|\boldsymbol{n} \cdot \boldsymbol{u}|_{\Gamma}=0\right\} .
$$


We also define the spaces whose functions are weakly divergence-free,

$$
\boldsymbol{J}_{0}:=\left\{\boldsymbol{u} \in \boldsymbol{L}^{2}(\Omega)|\nabla \cdot \boldsymbol{u}=0, \boldsymbol{n} \cdot \boldsymbol{u}|_{\Gamma}=0\right\},
$$

and

$$
\boldsymbol{J}_{1}:=\left\{\boldsymbol{u} \in \boldsymbol{H}_{0}^{1}(\Omega) \mid \nabla \cdot \boldsymbol{u}=0\right\} .
$$

Since $\boldsymbol{J}_{0}$ is a closed subspace of $\boldsymbol{L}^{2}(\Omega)$, this space can be decomposed as $\boldsymbol{L}^{2}(\Omega)=$ $\boldsymbol{J}_{0} \oplus \boldsymbol{J}_{0}^{\perp}$, where

$$
\boldsymbol{J}_{0}^{\perp}:=\left\{\boldsymbol{u} \in \boldsymbol{L}^{2}(\Omega) \mid \boldsymbol{u}=\nabla p, p \in \boldsymbol{H}^{1}(\Omega)\right\} .
$$

We define $P_{\boldsymbol{J}_{0}}$ as the orthogonal $\boldsymbol{L}^{2}(\Omega)$-projector onto $\boldsymbol{J}_{0}$. This operator, known as the Leray operator, is of main importance, and the basis of the original projection methods (see [4] and [26]). It is obviously continuous in $L^{2}(\Omega)$ by its definition. In fact, for $\Omega$ being an open bounded set of class $\mathcal{C}^{r+1}$, being $r$ an integer $\geq 1$, the operator $P_{J_{0}}$ also maps $H^{r}(\Omega)$ into itself and is continuous with respect to $\|\cdot\|_{r}$ (see [28]), that is to say, there exists a constant $C>0$ such that:
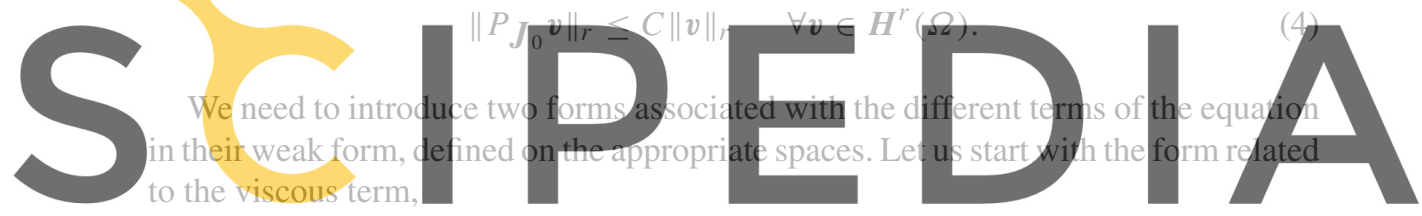

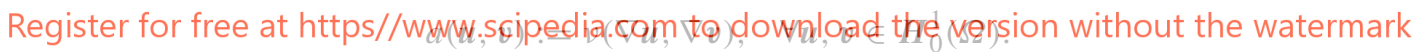

This is a bilinear continuous form on $\boldsymbol{H}_{0}^{1}(\Omega)$, which is coercive with respect to $\|\cdot\|_{1}$.

The second form to be introduced is required for the pressure gradient and the incompressibility constraint,

$$
b(\boldsymbol{v}, q):=-(q, \nabla \cdot \boldsymbol{v}), \quad \forall \boldsymbol{v} \in \boldsymbol{H}_{0}^{1}(\Omega), \forall q \in L^{2}(\Omega)
$$

It is also continuous with respect to the norms $\|q\|_{0}$ and $\|\boldsymbol{v}\|_{1}$.

Finally we introduce the trilinear form associated to the convective term in its standard form,

$$
c(\boldsymbol{u}, \boldsymbol{v}, \boldsymbol{w})=\int_{\Omega}(\boldsymbol{u} \cdot \nabla \boldsymbol{v}) \cdot \boldsymbol{w}, \quad \forall \boldsymbol{u}, \boldsymbol{v} \in \boldsymbol{H}^{1}(\Omega), \forall \boldsymbol{w} \in \boldsymbol{H}_{0}^{1}(\Omega)
$$

which is also continuous and well defined on these spaces. If $\boldsymbol{u} \in \boldsymbol{J}_{1}$, the form is skew-symmetric in its two last arguments,

$$
c(\boldsymbol{u}, \boldsymbol{v}, \boldsymbol{v})=0, \quad \forall \boldsymbol{u} \in \boldsymbol{J}_{1}, \forall \boldsymbol{v} \in \boldsymbol{H}_{0}^{1}(\Omega)
$$


Let us introduce some continuity properties that will be used hereafter, (see [10]):

$$
c(\boldsymbol{u}, \boldsymbol{v}, \boldsymbol{w}) \leq\left\{\begin{array}{l}
C\|\boldsymbol{u}\|_{1}\|\boldsymbol{v}\|_{1}\|\boldsymbol{w}\|_{1}, \\
C\|\boldsymbol{u}\|_{0}\|\boldsymbol{v}\|_{2}\|\boldsymbol{w}\|_{1}, \\
C\|\boldsymbol{u}\|_{2}\|\boldsymbol{v}\|_{1}\|\boldsymbol{w}\|_{0}, \\
C\|\boldsymbol{u}\|_{0}\|\boldsymbol{v}\|_{1}\|\boldsymbol{w}\|_{2}, \\
C\|\boldsymbol{u}\|_{2}\|\boldsymbol{v}\|_{0}\|\boldsymbol{w}\|_{1}
\end{array}\right.
$$

The skew-symmetric form, introduced by Temam in [28],

$$
\tilde{c}(\boldsymbol{u}, \boldsymbol{v}, \boldsymbol{w})=\frac{1}{2}(c(\boldsymbol{u}, \boldsymbol{v}, \boldsymbol{w})-c(\boldsymbol{u}, \boldsymbol{w}, \boldsymbol{v}))
$$

$\forall \boldsymbol{u} \in \boldsymbol{H}^{1}(\Omega), \boldsymbol{v}, \boldsymbol{w} \in \boldsymbol{H}_{0}^{1}(\Omega)$, is also used in the following. The previous boundedness properties of $c$ are also inherited by $\tilde{c}$. In any case the symbol $\sim$ will be omitted.

For the treatment of evolutionary problems, we require the following notation. Given $T>0,1 \leq p<\infty$ and $X$ a Banach space with norm $\|\cdot\|_{X}$, let $L^{p}(0, T ; X)$ be the space of functions $f:(0, T) \rightarrow X$ such that $\|f\|_{L p(0, T ; X)}=\left(\int_{0}^{T}\|f(s)\|_{X}^{p} d s\right)^{1 / p}<\infty$. In the case of $p=\infty$, we demand the property $\sup _{0 \leq s \leq T}$

\section{In addition, we defor}
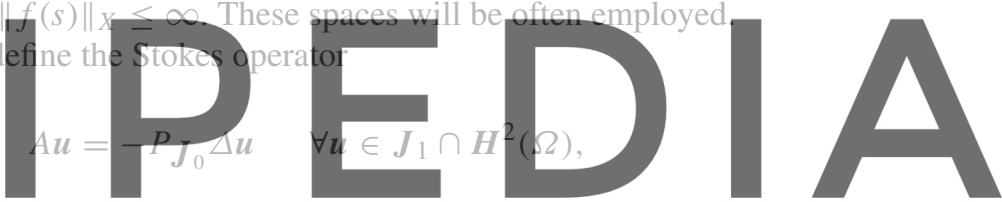

which is an unbounded positive self-adjoint closed operator onto $J_{0}$. The operator

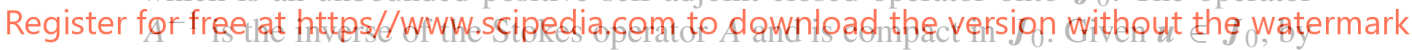

definition of $A, z=A^{-1} u$ is the solution of the following Stokes problem:

$$
\begin{aligned}
-\Delta z+\nabla \xi=\boldsymbol{u} & \text { in } \Omega, \\
\nabla \cdot z=0 & \text { in } \Omega, \\
z=\mathbf{0} & \text { on } \partial \Omega .
\end{aligned}
$$

When $\Omega$ is of class $\mathcal{C}^{2}$, or is a convex polygon or polyhedron (see [18]), there exists a constant $C>0$ such that

$$
\left\|A^{-1} \boldsymbol{u}\right\|_{s} \leq C\|\boldsymbol{u}\|_{s-2} \quad \text { for } s=1,2
$$

Furthermore, from (5) it follows that $\left(A^{-1} \boldsymbol{u}, \boldsymbol{u}\right)=\left\|A^{-1} \boldsymbol{u}\right\|_{1}^{2}$, and then it is easily seen that

$$
\|\boldsymbol{u}\|_{\boldsymbol{J}_{1}^{\prime}}^{2}=\left(A^{-1} \boldsymbol{u}, \boldsymbol{u}\right)
$$

for all $\boldsymbol{u} \in \boldsymbol{J}_{0}$ (remember that $\|\nabla \boldsymbol{u}\|_{0}$ is identified with the norm in $\boldsymbol{H}_{0}^{1}(\Omega)$ ). 
Assuming $\boldsymbol{u}_{0} \in \boldsymbol{H}_{0}^{1}$ and $\boldsymbol{f} \in L^{2}\left(0, T ; \boldsymbol{H}^{-1}(\Omega)\right)$, and if $\Omega$ is bounded and Lipschitz continuous, problem (1) has at least one solution $\boldsymbol{u} \in L^{\infty}\left(0, T ; \boldsymbol{L}^{2}(\Omega)\right) \cap$ $L^{2}\left(0, T ; \boldsymbol{H}_{0}^{1}(\Omega)\right)($ see $[28])$.

For the convergence analysis in this paper we assume more regularity of the solution. We assume that $\boldsymbol{u}(t)$ and $p(t)$ satisfy

(R1) $\boldsymbol{u}(t) \in \mathcal{C}\left(0, T ; \boldsymbol{J}_{1}\right) \cap L^{\infty}\left(0, T ; \boldsymbol{H}^{2}(\Omega)\right), p \in L^{\infty}\left(0, T ; H^{1}(\Omega)\right) \cap \mathcal{C}\left(0, T ; L^{2}\right.$ $(\Omega) / \mathbb{R})$

(R2a) $\tilde{\boldsymbol{u}} \in L^{\infty}\left(0, T ; \boldsymbol{L}^{2}(\Omega)\right)$

(R2b) $\tilde{\boldsymbol{u}} \in L^{2}\left(0, T ; \boldsymbol{H}_{0}^{1}(\Omega)\right)$.

Assumptions (R1), (R2a) and (R2b) can be proven assuming for the data

$$
\boldsymbol{u}_{0} \in \boldsymbol{H}^{2}(\Omega) \cap \boldsymbol{J}_{1}, \quad \boldsymbol{f}(t) \in L^{\infty}\left(0, T ; \boldsymbol{L}^{2}(\Omega)\right) \cap L^{2}\left(0, T ; \boldsymbol{H}^{1}(\Omega)\right),
$$

and if $d=3$, we also need to assume

$$
u(t) \in L^{\infty}\left(0, T ; H^{1}(\Omega)\right),
$$

as it is proved in [18]. The last assumption can be replaced by a condition that is satisfied if $\nu$ is large enough or if $f(t)$ and $u_{0}$ are small enough for a $\Omega$ of class $C^{2}$ (see [28, Theorems 3.7 and 3.87). The next assumption will be needed in the convergence analys Assumption 1 Co
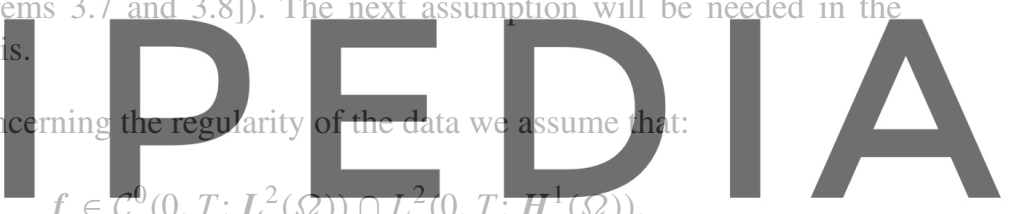

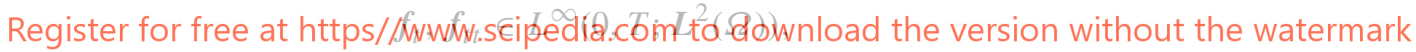
$u_{0} \in H^{2}(\Omega) \cap J_{1}$,

and, if $d=3$,

$$
\boldsymbol{u}(t) \in \boldsymbol{L}^{\infty}\left(0, T ; \boldsymbol{H}^{1}(\Omega)\right) .
$$

This implies the regularity properties (R1), (R2a) and (R2b). Furthermore, the following assumption concerning the regularity of the domain $\Omega$ is used,

Assumption 2 The inverse of the Stokes operator $A^{-1}$ verifies the regularity property (6), with $s=2$.

If $\Omega$ is of class $\mathcal{C}^{2}$ this assumption is satisfied.

In order to avoid any incompatible condition as $t \rightarrow 0$, let us introduce the weighting function $\lambda:=\min \left\{t, t_{0}\right\}$ and its discrete counterpart $\lambda_{n}:=\min \left\{t^{n}, t_{0}\right\}$, where $t_{0}>0$ is fixed. It allows us to obtain error estimates without taking care of what happens near $t=0$ (see [22]).

To define the order of approximation of time integration schemes for time-dependent partial differential equations, the following terminology is used: Given a Banach space 
$X$ equipped with $\|\cdot\|_{X}$, a continuous function $f:[0, T] \longrightarrow X$ and two real numbers $0<p<\infty$ and $\alpha>0$, for a time step size $\Delta t>0$, let $t^{n}=n \Delta t$ for $n=0, \ldots, N=[T / \Delta t]-1$. A sequence $\left\{f^{n}\right\}_{n=1}^{N}$ is said to be an $\alpha$-th order approximation of $f$ in $\ell^{p}(X)$ if there exists a constant $C$ independent of $\Delta t$ such that

$$
\left(\sum_{n=1}^{N} \Delta t\left\|f\left(t^{n+1}\right)-f^{n+1}\right\|_{X}^{p}\right)^{1 / p}<C \Delta t^{\alpha} .
$$

Moreover, $\left\{f^{n}\right\}_{n=1}^{N}$ is said to be an $\alpha$-th order approximation of $f$ in $\ell^{\infty}(X)$ if there exists a constant $C$ independent of $\Delta t$ and $n$ such that

$$
\left\|f\left(t^{n+1}\right)-f^{n+1}\right\|_{X}<C \Delta t^{\alpha} \quad \forall n=0, \ldots, N .
$$

For the stability result we will use the skew-symmetric (in its last two arguments) part of the convective form $c$ in the analysis of the fully discrete problem.

From here onwards $C$ denotes a positive constant, not necessarily the same at different appearances. Moreover, we denote by $N=[T / \Delta t]-1$.

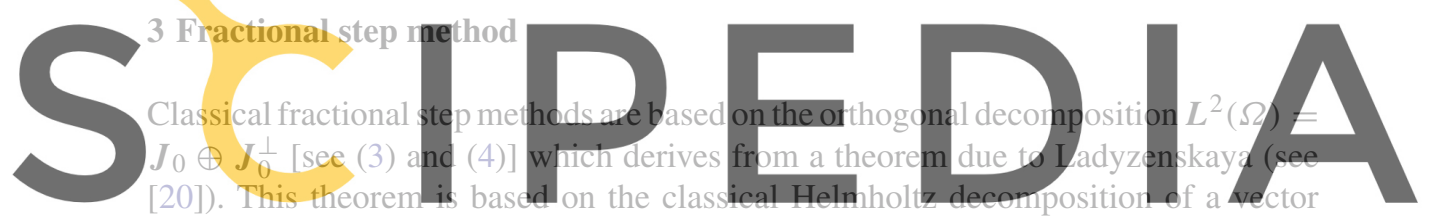

field into the sum of a solenoidal field and a gradient of a scalar function and a more

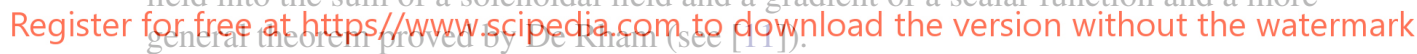

Hence, in the projection method an intermediate velocity obtained from the momentum equation without the pressure term is decomposed into a solenoidal field, the velocity, and the gradient of a scalar field, the pressure. We present this method in its semi-discrete version in time.

This procedure involves two basic steps. The first step consists of finding an intermediate velocity $\hat{\boldsymbol{u}}^{n+1}$ such that:

$$
\begin{aligned}
\frac{1}{\Delta t}\left(\hat{\boldsymbol{u}}^{n+1}-\boldsymbol{u}^{n}\right)-v \Delta \hat{\boldsymbol{u}}^{n+1}+\hat{\boldsymbol{u}}^{n+1} \cdot \nabla \hat{\boldsymbol{u}}^{n+1} & =\boldsymbol{f}^{n+1}, \\
\left.\hat{\boldsymbol{u}}^{n+1}\right|_{\Gamma} & =\mathbf{0} .
\end{aligned}
$$

In (7a) the convective term is treated implicitly. Alternatively, explicit and semiimplicit approaches can be used. Note that the full Dirichlet boundary conditions are imposed on $\hat{\boldsymbol{u}}^{n+1}$. The weak form of this equation is required for the numerical approximation. Its weak formulation consists of: find $\hat{\boldsymbol{u}}^{n+1} \in \boldsymbol{H}_{0}^{1}(\Omega)$ such that

$$
\frac{1}{\Delta t}\left(\hat{\boldsymbol{u}}^{n+1}-\boldsymbol{u}^{n}, \boldsymbol{v}\right)+a\left(\hat{\boldsymbol{u}}^{n+1}, \boldsymbol{v}\right)+c\left(\hat{\boldsymbol{u}}^{n+1}, \hat{\boldsymbol{u}}^{n+1}, \boldsymbol{v}\right)=\left\langle\boldsymbol{f}^{n+1}, \boldsymbol{v}\right\rangle
$$


$\forall \boldsymbol{v} \in \boldsymbol{H}_{0}^{1}(\Omega)$. Temam [28] proposed the use of the skew-symmetric form of the convective term in (8). This form is usually assumed in order to obtain stability bounds for the solution of implicit projection methods.

The second step of the method consists of finding an end-of-step velocity $\boldsymbol{u}^{n+1}$ and a pressure $p^{n+1}$ such that

$$
\begin{aligned}
\frac{1}{\Delta t}\left(\boldsymbol{u}^{n+1}-\hat{\boldsymbol{u}}^{n+1}\right)+\nabla p^{n+1} & =\mathbf{0}, \\
\nabla \cdot \boldsymbol{u}^{n+1} & =0, \\
\left.\boldsymbol{n} \cdot \boldsymbol{u}^{n+1}\right|_{\Gamma} & =0,7
\end{aligned}
$$

which is equivalent to find the projection of $\hat{\boldsymbol{u}}^{n+1}$ onto the space $\boldsymbol{J}_{0}$ [see (2)],

$$
\boldsymbol{u}^{n+1}=P_{\boldsymbol{J}_{0}}\left(\hat{\boldsymbol{u}}^{n+1}\right) \text {. }
$$

We point out that at this step only the normal component of the velocity is prescribed. Then, the end-of-step velocity $\boldsymbol{u}^{n+1}$ does not satisfy the proper boundary conditions. Hence, we infereasily from (7b) and (9c) that the pressure is satisfying on the Dirichlet boundary an artificial Neumann condition,

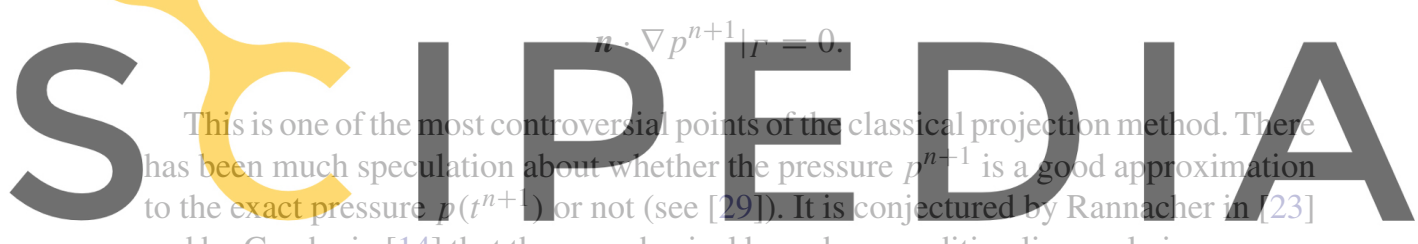

and by Gresho in [14] that the non-physical boundary condition lives only in a narrow

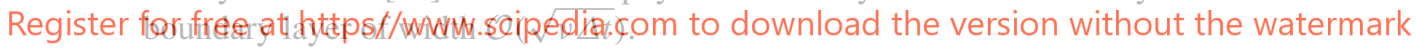

The main interest of the projection method is the possible uncoupling of the pressure from the velocity in its numerical approximation. It is achieved by taking the divergence of (9a), obtaining a Pressure Poisson Equation for the pressure,

$$
\begin{aligned}
\Delta p^{n+1} & =\frac{1}{\delta t} \nabla \cdot \hat{\boldsymbol{u}}^{n+1}, \\
\left.\boldsymbol{n} \cdot \nabla p^{n+1}\right|_{\Gamma} & =0 .
\end{aligned}
$$

Once the pressure $p^{n+1}$ is calculated, the end-of-step velocity is recovered from (9a).

\section{Analysis of a semi-discrete projection-like problem}

In this section we list some optimal convergence results and stability results for an auxiliary problem that can be identified as a semi-discrete projection method evaluating exactly the convective term. The proof of these results is fairly standard. We refer to [1] for the detailed analysis of this auxiliary problem that has not been included here for the sake of brevity. In fact, these results have been obtained pursuing the analysis carried out by Prohl in [22] for the semi-discrete projection method with the only 
diference that now the convective term does not introduce any error to the system. In order to obtain strong stability bounds in Lemma 1, special attention has been paid for the regularity of the semi-discrete derivatives.

Let us start introducing the auxiliary problem at the continuous level. It consists of finding $\hat{\boldsymbol{w}}^{n+1} \in \boldsymbol{H}_{0}^{1}(\Omega), \boldsymbol{w}^{n+1} \in \boldsymbol{H}(\operatorname{div}, \Omega)$ and $\phi^{n+1} \in H_{0}^{1}(\Omega) / \mathbb{R}$ such that,

$$
\begin{aligned}
\frac{1}{\delta t}\left(\hat{\boldsymbol{w}}^{n+1}-\boldsymbol{w}^{n}\right)-v \Delta \hat{\boldsymbol{w}}^{n+1}+\boldsymbol{u}\left(t^{n+1}\right) \cdot \nabla \boldsymbol{u}\left(t^{n+1}\right) & =\boldsymbol{f}\left(t^{n+1}\right), \\
\frac{1}{\delta t}\left(\boldsymbol{w}^{n+1}-\hat{\boldsymbol{w}}^{n+1}\right)+\nabla \phi^{n+1} & =\mathbf{0}, \\
\nabla \cdot \boldsymbol{w}^{n+1} & =0,
\end{aligned}
$$

with boundary conditions

$$
\begin{array}{r}
\left.\hat{\boldsymbol{w}}^{n+1}\right|_{\Gamma}=\mathbf{0}, \\
\left.\boldsymbol{n} \cdot \boldsymbol{w}^{n+1}\right|_{\Gamma}=0 .
\end{array}
$$

We characterize the projection-like problem with some estimates for the auxiliary unknowns. But first Assumption 3 Expression

If $\Omega$ is of class $C^{3}$ this assumption and Assumption 2 are satisfied.

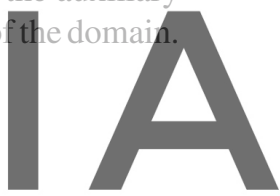

Register for free at https//www.scipedia.com to download the version without the watermark Lemma 1 (Stability) Under Assumptions 1 and 2, the following stability results hold

$$
\max _{0 \leq n \leq N}\left\{\left\|\hat{\boldsymbol{w}}^{n+1}\right\|_{2}^{2}+\left\|\nabla \phi^{n+1}\right\|_{0}^{2}+\left\|\frac{1}{\delta t}\left(\boldsymbol{w}^{n+1}-\hat{\boldsymbol{w}}^{n+1}\right)\right\|_{0}\right\} \leq C,
$$

for $\Delta t>0$ small enough. Furthermore, under Assumption 3

$$
\max _{0 \leq n \leq N}\left\|\boldsymbol{w}^{n+1}\right\|_{2} \leq C
$$

Remark 1 These sharp stability bounds are a key feature of our auxiliary problem and the justification of its introduction. In the convergence analysis of the fully discrete method developed later these properties will be exploited in order to obtain a bound for the error related to the convective term.

We end the section with a convergence result for the projection-like system. This result is basic for the obtention of convergence results for the fully discrete projection method. 
Corollary 1 (Convergence) Under Assumptions 1, 2 together with the regularity assumption $\partial_{t}^{3} \boldsymbol{f}(t) \in L^{\infty}\left(0, T ; \boldsymbol{L}^{2}(\Omega)\right)$, the following convergence results hold,

$$
\begin{gathered}
\max _{0 \leq n \leq N}\left\{\left\|\boldsymbol{u}\left(t^{n+1}\right)-\hat{\boldsymbol{w}}^{n+1}\right\|_{0}+\left\|\boldsymbol{u}\left(t^{n+1}\right)-\boldsymbol{w}^{n+1}\right\|_{0}\right\} \leq C \Delta t, \\
\max _{0 \leq n \leq N}\left\{\left\|\boldsymbol{u}\left(t^{n+1}\right)-\hat{\boldsymbol{w}}^{n+1}\right\|_{1}+\lambda_{n+1}\left\|p\left(t^{n+1}\right)-\phi^{n+1}\right\|_{0}\right\} \leq C \sqrt{\Delta t},
\end{gathered}
$$

for $\Delta t>0$ small enough.

\section{Convergence results satisfying the inf-sup condition}

In this section we obtain optimal convergence results for the projection method with a velocity-pressure interpolation satisfying the inf-sup condition. Furthermore, the end-of-step velocity and the pressure are solved in a coupled way. It implies that the end-of-step velocity is weakly divergence free. The classical first order fractional step method, also called projection method, in its fully discretized version reads as follows: find the solution $\left(\hat{\boldsymbol{u}}_{h}^{n+1}, \boldsymbol{u}_{h}^{n+1}, p_{h}^{n+1}\right) \in \mathcal{V}_{h, 0} \times \mathcal{Y}_{h, 0} \times \mathcal{Q}_{h}$ such that,

$$
\begin{array}{ll}
\frac{1}{\delta t}\left(\hat{\boldsymbol{u}}_{h}^{n+1}-\boldsymbol{u}_{h}^{n}, \boldsymbol{v}_{h}\right)+v\left(\nabla \hat{\boldsymbol{u}}_{h}^{n+1}, \nabla \boldsymbol{v}_{h}\right)+c\left(\hat{\boldsymbol{u}}_{h}^{n+1}, \hat{\boldsymbol{u}}_{h}^{n+1}, \boldsymbol{v}_{h}\right)=\left\langle\boldsymbol{f}\left(t^{n+1}\right), \boldsymbol{v}_{h}\right\rangle, \forall \boldsymbol{v}_{h} \in \mathcal{V}_{h, 0} \\
\frac{1}{\delta t}\left(\boldsymbol{u}_{h}^{n+1}-\hat{\boldsymbol{u}}_{h}^{n+1}, \boldsymbol{y}_{h}\right)+\left(\nabla p_{h}^{n+1}, \boldsymbol{y}_{h}\right)=0, & \forall \boldsymbol{y}_{h} \in \mathcal{Y}_{h, 0}, \\
\left(\nabla \cdot \boldsymbol{u}_{h}^{n+1}, q_{h}\right)=0, & \forall q_{h} \in \mathcal{Q}_{h} .
\end{array}
$$

where $\mathcal{V}_{h, 0}, \mathcal{Y}_{h, 0}, \mathcal{Q}_{h}$ are classical finite element approximation spaces of $\boldsymbol{H}_{0}^{1}(\Omega)$, $\boldsymbol{H}_{0}(\operatorname{div}, \Omega)$ and $L^{2}(\Omega) / \mathbb{R}$ (see [3]). Let us also consider $\mathcal{V}_{h}$ a finite element approximation of $\boldsymbol{H}^{1}(\Omega)$.

For the analysis in this section we need Assumptions 1 and 2 stated in the previous section and the additional assumptions on the space discretization listed below.

Let us denote by $B_{h}$ the discrete operator

$$
B_{h}: \mathcal{V}_{h, 0} \rightarrow \mathcal{Q}_{h}^{\prime} \quad \mid\left\langle B_{h}\left(\boldsymbol{v}_{h}\right), q_{h}\right\rangle_{\mathcal{Q}_{h}^{\prime} \times \mathcal{Q}_{h}}=b\left(\boldsymbol{v}_{h}, q_{h}\right)
$$

$\forall \boldsymbol{v} \in \mathcal{V}_{h, 0}$ and $\forall q_{h} \in \mathcal{Q}_{h}$, where $\mathcal{Q}_{h}^{\prime}$ is the dual space of $\mathcal{Q}_{h}$.

Assumption 4 There exists a constant $\beta_{d}$ independent of the mesh size $h$ such that

$$
\inf _{q_{h} \in \mathcal{Q}_{h}} \sup _{\boldsymbol{v}_{h} \in \mathcal{V}_{h, 0}} \frac{b\left(\boldsymbol{v}_{h}, q_{h}\right)}{\left\|\boldsymbol{v}_{h}\right\| \mathcal{V}_{h, 0}\left\|q_{h}\right\|_{\mathcal{Q}_{h} / \operatorname{ker} B_{h}^{t}}} \geq \beta_{d}>0 .
$$

We are relying on the inf-sup condition for the analysis of this section. 
Assumption 5 The discrete space $\mathcal{V}_{h, 0}$ is a subspace of $\mathcal{Y}_{h, 0}$, so that

$$
\mathcal{V}_{h, 0} \subseteq \mathcal{Y}_{h, 0}
$$

Assumption 6 The family of finite element partitions $\left\{\Theta_{h}\right\}_{h>0}$ is quasi-uniform.

This assumption is needed because if it holds the following inverse estimate (see [3]) can be used: given $\boldsymbol{v}_{h} \in \mathcal{V}_{h, 0}$, there exists a constant $C$ independent of the mesh size $h$ such that

$$
\left\|\boldsymbol{v}_{h}\right\|_{1} \leq \frac{C}{h}\left\|\boldsymbol{v}_{h}\right\|_{0}
$$

Assumption 7 There exists a constant $C>0$ independent of $h$ and $\Delta t$ such that

$$
\Delta t \geq C h^{2} \text {. }
$$

The proofs of convergence results for this method rely on the auxiliary problem proposed and analyzed in the previous section. We compare the solution of the fully discrete projection method with the solution of the auxiliary problem for which we have optimal convergence results.

Let us denote the discrete velocity and pressure errors:

$$
\begin{aligned}
\hat{\boldsymbol{e}}_{d}^{n+1} & =\hat{\boldsymbol{w}}^{n+1}-\hat{\boldsymbol{u}}_{h}^{n+1}, \\
\boldsymbol{e}_{d}^{n+1} & =\boldsymbol{w}^{n+1}-\boldsymbol{u}_{h}^{n+1}, \\
r_{d}^{n+1} & =\phi^{n+1}-p_{h}^{n+1},
\end{aligned}
$$

that are governed by the system obtained subtracting (12) from (11),

$$
\begin{aligned}
\frac{1}{\delta t}\left(\hat{\boldsymbol{e}}_{d}^{n+1}-\boldsymbol{e}_{d}^{n}, \boldsymbol{v}_{h}\right)+v\left(\nabla \hat{\boldsymbol{e}}_{d}^{n+1}, \nabla \boldsymbol{v}_{h}\right) & =c\left(\hat{\boldsymbol{u}}_{h}^{n+1}, \hat{\boldsymbol{u}}_{h}^{n+1}, \boldsymbol{v}_{h}\right)-c\left(\boldsymbol{u}\left(t^{n+1}\right), \boldsymbol{u}\left(t^{n+1}\right), \boldsymbol{v}_{h}\right) \\
\frac{1}{\delta t}\left(\boldsymbol{e}_{d}^{n+1}-\hat{\boldsymbol{e}}_{d}^{n+1}, \boldsymbol{y}_{h}\right)+\left(\nabla r_{d}^{n+1}, \boldsymbol{y}_{h}\right) & =0, \\
\left(\nabla \cdot \boldsymbol{e}_{d}^{n+1}, q_{h}\right) & =0 .
\end{aligned}
$$

Likewise, we introduce the continuous velocity errors:

$$
\begin{aligned}
& \hat{\boldsymbol{e}}_{c}^{n+1}=\boldsymbol{u}\left(t^{n+1}\right)-\hat{\boldsymbol{w}}^{n+1}, \\
& \boldsymbol{e}_{c}^{n+1}=\boldsymbol{u}\left(t^{n+1}\right)-\boldsymbol{w}^{n+1} .
\end{aligned}
$$

We also use the following notation for the interpolation error:

$$
\begin{aligned}
\tilde{\mathcal{I}}_{n}(h)= & \frac{1}{h} \inf _{\boldsymbol{v}_{h} \in \mathcal{V}_{h, 0}}\left\|\hat{\boldsymbol{w}}^{n+1}-\boldsymbol{v}_{h}\right\|_{0}+\frac{1}{h} \inf _{\boldsymbol{y}_{h} \in \mathcal{Y}_{h, 0}}\left\|\boldsymbol{w}^{n+1}-\boldsymbol{y}_{h}\right\|_{0} \\
& +\inf _{\boldsymbol{v}_{h} \in \mathcal{V}_{h, 0}}\left\|\hat{\boldsymbol{w}}^{n+1}-\boldsymbol{v}_{h}\right\|_{1}+\inf _{q_{h} \in \mathcal{Q}_{h}}\left\|\phi^{n+1}-q_{h}\right\|_{0}
\end{aligned}
$$


Remark 2 We note that the interpolation error introduced is related to the solution of the auxiliary problem. This is optimal in the case of div-stable first order elements, e.g. the $P_{1}^{+}-P_{1}$ mini-element. When using higher order elements, as the $P_{2}-P_{1}$, further regularity on the semi-discrete unknown would allow to improve the overall convergence rate.

Under the discrete inf-sup condition, optimal order approximations of so-lenoidal vector fields can be achieved by means of discretely divergence free finite element functions, that is, $x_{h} \in \operatorname{ker} B_{h}$. In fact, one has the following result (see [13]).

Lemma 2 Let $\boldsymbol{u} \in \boldsymbol{J}_{1}$ and assume that discrete spaces $\mathcal{V}_{h, 0}$ and $\mathcal{Q}_{h}$ satisfy Assumption 4 and 6. Then,

$$
\inf _{\boldsymbol{x}_{h} \in \operatorname{ker} B_{h}}\left\|\boldsymbol{u}-\boldsymbol{x}_{h}\right\|_{1} \leq C \inf _{\boldsymbol{v}_{h} \in \mathcal{V}_{h, 0}}\left\|\boldsymbol{u}-\boldsymbol{v}_{h}\right\|_{1}
$$

Moreover, under Assumption 2 we also have,

$$
\inf _{\boldsymbol{x}_{h} \in \operatorname{ker} B_{h}}\left\|\boldsymbol{u}-\boldsymbol{x}_{h}\right\|_{0} \leq C h \inf _{\boldsymbol{v}_{h} \in \mathcal{V}_{h, 0}}\left\|\boldsymbol{u}-\boldsymbol{v}_{h}\right\|_{1} .
$$

A first error estimate showing that $\left\{\hat{\boldsymbol{u}}_{h}^{n+1}\right\}_{n=0, \ldots, N}$ and $\left\{\boldsymbol{u}_{h}^{n+1}\right\}_{n=0, \ldots, N}$ are first order approximations to $\left\{\boldsymbol{u}\left(t^{n+1}\right)\right\}_{n=0, \ldots, N}$ with respect to the norm associated to $\ell^{\infty}\left(\boldsymbol{L}^{2}(\Omega)\right) \cap \ell^{2}\left(\boldsymbol{H}^{1}(\Omega)\right)$ is established in the following theorem.

Theorem 1 Being valid Assumptions 1, 2, 4, 5, 6 and 7, we have

$$
\begin{aligned}
& \max _{0 \leq n \leq N}\left\{\left\|\boldsymbol{e}_{d}^{N+1}\right\|_{0}^{2}+\left\|\hat{\boldsymbol{e}}_{d}^{N+1}\right\|_{0}^{2}\right\}+\sum_{n=0}^{N} v \Delta t\left\|\hat{\boldsymbol{e}}_{d}^{n+1}\right\|_{1}^{2} \\
& +\sum_{n=0}^{N}\left\{\left\|\boldsymbol{e}_{d}^{n+1}-\hat{\boldsymbol{e}}_{d}^{n+1}\right\|_{0}^{2}+\left\|\hat{\boldsymbol{e}}_{d}^{n+1}-\boldsymbol{e}_{d}^{n+1}\right\|_{0}^{2}\right\} \\
& \quad+\sum_{n=0}^{N} \Delta t^{2}\left\|r_{d}^{n+1}\right\|_{0}^{2} \leq C\left(\tilde{\mathcal{I}}(h)^{2}+\Delta t^{2}\right),
\end{aligned}
$$

for $\Delta t>0$ small enough.

Proof Taking $\Delta t\left(\boldsymbol{v}_{h}-\hat{\boldsymbol{u}}_{h}^{n+1}\right)$ as a test function in (14a), we obtain

$$
\begin{aligned}
& \frac{1}{2}\left(\left\|\hat{\boldsymbol{e}}_{d}^{n+1}\right\|_{0}^{2}-\left\|\boldsymbol{e}_{d}^{n}\right\|_{0}^{2}+\left\|\hat{\boldsymbol{e}}_{d}^{n+1}-\boldsymbol{e}_{d}^{n}\right\|_{0}^{2}\right)+v \Delta t\left\|\hat{\boldsymbol{e}}_{d}^{n+1}\right\|_{1}^{2} \\
& =\Delta t c\left(\hat{\boldsymbol{u}}_{h}^{n+1}, \hat{\boldsymbol{u}}_{h}^{n+1}, \boldsymbol{v}_{h}-\hat{\boldsymbol{u}}_{h}^{n+1}\right)-\Delta t c\left(\boldsymbol{u}\left(t^{n+1}\right), \boldsymbol{u}\left(t^{n+1}\right), \boldsymbol{v}_{h}-\hat{\boldsymbol{u}}_{h}^{n+1}\right) \\
& \quad+\left(\hat{\boldsymbol{e}}_{d}^{n+1}-\boldsymbol{e}_{d}^{n}, \hat{\boldsymbol{w}}^{n+1}-\boldsymbol{v}_{h}\right)+v \Delta t\left(\nabla \hat{\boldsymbol{e}}_{d}^{n+1}, \nabla\left(\hat{\boldsymbol{w}}^{n+1}-\boldsymbol{v}_{h}\right)\right) .
\end{aligned}
$$


We combine the nonlinear terms as follows:

$$
\begin{aligned}
- & c\left(\boldsymbol{u}\left(t^{n+1}\right), \boldsymbol{u}\left(t^{n+1}\right), \boldsymbol{v}_{h}-\hat{\boldsymbol{u}}_{h}^{n+1}\right)+c\left(\hat{\boldsymbol{u}}_{h}^{n+1}, \hat{\boldsymbol{u}}_{h}^{n+1}, \boldsymbol{v}_{h}-\hat{\boldsymbol{u}}_{h}^{n+1}\right) \\
= & -c\left(\boldsymbol{u}\left(t^{n+1}\right), \hat{\boldsymbol{e}}_{c}^{n+1}, \boldsymbol{v}_{h}-\hat{\boldsymbol{u}}_{h}^{n+1}\right)-c\left(\hat{\boldsymbol{u}}_{h}^{n+1}, \hat{\boldsymbol{e}}_{d}^{n+1}, \boldsymbol{v}_{h}-\hat{\boldsymbol{u}}_{h}^{n+1}\right) \\
& -c\left(\hat{\boldsymbol{e}}_{c}^{n+1}, \hat{\boldsymbol{w}}^{n+1}, \boldsymbol{v}_{h}-\hat{\boldsymbol{u}}_{h}^{n+1}\right)-c\left(\hat{\boldsymbol{e}}_{d}^{n+1}, \hat{\boldsymbol{w}}^{n+1}, \boldsymbol{v}_{h}-\hat{\boldsymbol{u}}_{h}^{n+1}\right) \\
= & -c\left(\boldsymbol{u}\left(t^{n+1}\right), \hat{\boldsymbol{e}}_{c}^{n+1}, \hat{\boldsymbol{e}}_{d}^{n+1}\right)+c\left(\boldsymbol{u}\left(t^{n+1}\right), \hat{\boldsymbol{e}}_{c}^{n+1}, \hat{\boldsymbol{w}}^{n+1}-\boldsymbol{v}_{h}\right)-c\left(\hat{\boldsymbol{w}}^{n+1}, \hat{\boldsymbol{e}}_{d}^{n+1}, \hat{\boldsymbol{e}}_{d}^{n+1}\right) \\
& +c\left(\hat{\boldsymbol{w}}^{n+1}, \hat{\boldsymbol{e}}_{d}^{n+1}, \hat{\boldsymbol{w}}^{n+1}-\boldsymbol{v}_{h}\right)+c\left(\hat{\boldsymbol{e}}_{d}^{n+1}, \hat{\boldsymbol{e}}_{d}^{n+1}, \hat{\boldsymbol{e}}_{d}^{n+1}\right)-c\left(\hat{\boldsymbol{e}}_{d}^{n+1}, \hat{\boldsymbol{e}}_{d}^{n+1}, \hat{\boldsymbol{w}}^{n+1}-\boldsymbol{v}_{h}\right) \\
& -c\left(\hat{\boldsymbol{e}}_{c}^{n+1}, \hat{\boldsymbol{w}}^{n+1}, \hat{\boldsymbol{e}}_{d}^{n+1}\right)+c\left(\hat{\boldsymbol{e}}_{c}^{n+1}, \hat{\boldsymbol{w}}^{n+1}, \hat{\boldsymbol{w}}^{n+1}-\boldsymbol{v}_{h}\right) \\
& -c\left(\hat{\boldsymbol{e}}_{d}^{n+1}, \hat{\boldsymbol{w}}^{n+1}, \hat{\boldsymbol{e}}_{d}^{n+1}\right)+c\left(\hat{\boldsymbol{e}}_{d}^{n+1}, \hat{\boldsymbol{w}}^{n+1}, \hat{\boldsymbol{w}}^{n+1}-\boldsymbol{v}_{h}\right) .
\end{aligned}
$$

Every nonlinear term can be appropriately bounded as follows:

$$
\begin{aligned}
& -c\left(\boldsymbol{u}\left(t^{n+1}\right), \hat{\boldsymbol{e}}_{c}^{n+1}, \hat{\boldsymbol{e}}_{d}^{n+1}\right) \leq C\left\|\boldsymbol{u}\left(t^{n+1}\right)\right\|_{2}\left\|\hat{\boldsymbol{e}}_{c}^{n+1}\right\|_{0}\left\|\hat{\boldsymbol{e}}_{d}^{n+1}\right\|_{1} \\
& \leq C\left\|\hat{\boldsymbol{e}}_{c}^{n+1}\right\|_{0}^{2}+\frac{1}{8} v\left\|\hat{\boldsymbol{e}}_{d}^{n+1}\right\|_{1}^{2} \\
& \leq C \Delta t^{2}+\frac{1}{8} \nu\left\|\hat{\boldsymbol{e}}_{d}^{n+1}\right\|_{1}^{2}, \\
& c\left(\boldsymbol{u}\left(t^{n+1}\right), \hat{\boldsymbol{e}}_{c}^{n+1}, \hat{\boldsymbol{w}}^{n+1}-\boldsymbol{v}_{h}\right) \leq C\left\|\boldsymbol{u}\left(t^{n+1}\right)\right\|_{2}\left\|\hat{\boldsymbol{e}}_{c}^{n+1}\right\|_{0}\left\|\hat{\boldsymbol{w}}^{n+1}-\boldsymbol{v}_{h}\right\|_{1} \\
& \leq C\left\|\hat{\boldsymbol{e}}_{c}^{n+1}\right\|_{0}^{2}+C\left\|\hat{\boldsymbol{w}}^{n+1}-\boldsymbol{v}_{h}\right\|_{1}^{2} \\
& \leq C \Delta t^{2}+C\left\|\hat{\boldsymbol{w}}^{n+1}-\boldsymbol{v}_{h}\right\|_{1}^{2}, \\
& -c\left(\hat{\boldsymbol{w}}^{n+1}, \hat{\boldsymbol{e}}_{d}^{n+1}, \hat{\boldsymbol{e}}_{d}^{n+1}\right)=0, \\
& c\left(\hat{\boldsymbol{w}}^{n+1}, \hat{\boldsymbol{e}}_{d}^{n+1}, \hat{\boldsymbol{w}}^{n+1}-\boldsymbol{v}_{h}\right) \leq\left\|\hat{\boldsymbol{w}}^{n+1}\right\|_{1}\left\|\hat{\boldsymbol{e}}_{d}^{n+1}\right\|_{1}\left\|\hat{\boldsymbol{w}}^{n+1}-\boldsymbol{v}_{h}\right\|_{1} \\
& \leq \frac{1}{8} v\left\|\hat{\boldsymbol{e}}_{d}^{n+1}\right\|_{1}^{2}+C\left\|\hat{\boldsymbol{w}}^{n+1}-\boldsymbol{v}_{h}\right\|_{1}^{2}, \\
& c\left(\hat{\boldsymbol{e}}_{d}^{n+1}, \hat{\boldsymbol{e}}_{d}^{n+1}, \hat{\boldsymbol{e}}_{d}^{n+1}\right)=0, \\
& -c\left(\hat{\boldsymbol{e}}_{d}^{n+1}, \hat{\boldsymbol{e}}_{d}^{n+1}, \hat{\boldsymbol{w}}^{n+1}-\boldsymbol{v}_{h}\right) \leq C\left\|\hat{\boldsymbol{e}}_{d}^{n+1}\right\|_{1}\left\|\hat{\boldsymbol{e}}_{d}^{n+1}\right\|_{1}\left\|\hat{\boldsymbol{w}}^{n+1}-\boldsymbol{v}_{h}\right\|_{1} \\
& \leq \frac{1}{8} v\left\|\hat{\boldsymbol{e}}_{d}^{n+1}\right\|_{1}^{2}+C v\left\|\hat{\boldsymbol{e}}_{d}^{n+1}\right\|_{1}^{2}\left\|\hat{\boldsymbol{w}}^{n+1}-\boldsymbol{v}_{h}\right\|_{1}^{2}, \\
& -c\left(\hat{\boldsymbol{e}}_{c}^{n+1}, \hat{\boldsymbol{w}}^{n+1}, \hat{\boldsymbol{e}}_{d}^{n+1}\right) \leq C\left\|\hat{\boldsymbol{e}}_{c}^{n+1}\right\|_{0}\left\|\hat{\boldsymbol{w}}^{n+1}\right\|_{2}\left\|\hat{\boldsymbol{e}}_{d}^{n+1}\right\|_{1} \\
& \leq C\left\|\hat{\boldsymbol{e}}_{c}^{n+1}\right\|_{0}^{2}+\frac{1}{8} v\left\|\hat{\boldsymbol{e}}_{d}^{n+1}\right\|_{1}^{2} \\
& \leq C \Delta t^{2}+\frac{1}{8} v\left\|\hat{\boldsymbol{e}}_{d}^{n+1}\right\|_{1}^{2}, \\
& c\left(\hat{\boldsymbol{e}}_{c}^{n+1}, \hat{\boldsymbol{w}}^{n+1}, \hat{\boldsymbol{w}}^{n+1}-\boldsymbol{v}_{h}\right) \leq C\left\|\hat{\boldsymbol{e}}_{c}^{n+1}\right\|_{0}\left\|\hat{\boldsymbol{w}}^{n+1}\right\|_{2}\left\|\hat{\boldsymbol{w}}^{n+1}-\boldsymbol{v}_{h}\right\|_{1} \\
& \leq C\left\|\hat{\boldsymbol{e}}_{c}^{n+1}\right\|_{0}^{2}+C\left\|\hat{\boldsymbol{w}}^{n+1}-\boldsymbol{v}_{h}\right\|_{1}^{2} \\
& \leq C \Delta t^{2}+C\left\|\hat{\boldsymbol{w}}^{n+1}-\boldsymbol{v}_{h}\right\|_{1}^{2}, \\
& -c\left(\hat{\boldsymbol{e}}_{d}^{n+1}, \hat{\boldsymbol{w}}^{n+1}, \hat{\boldsymbol{e}}_{d}^{n+1}\right) \leq\left\|\hat{\boldsymbol{e}}_{d}^{n+1}\right\|_{0}\left\|\hat{\boldsymbol{w}}^{n+1}\right\|_{2}\left\|\hat{\boldsymbol{e}}_{d}^{n+1}\right\|_{1} \\
& \leq C\left\|\boldsymbol{e}_{d}^{n+1}\right\|_{0}^{2}+C\left\|\boldsymbol{e}_{d}^{n+1}-\hat{\boldsymbol{e}}_{d}^{n+1}\right\|_{0}^{2}+\frac{1}{8} \nu\left\|\hat{\boldsymbol{e}}_{d}^{n+1}\right\|_{1}^{2},
\end{aligned}
$$




$$
\begin{aligned}
c\left(\hat{\boldsymbol{e}}_{d}^{n+1}, \hat{\boldsymbol{w}}^{n+1}, \hat{\boldsymbol{w}}^{n+1}-\boldsymbol{v}_{h}\right) & \leq\left\|\hat{\boldsymbol{e}}_{d}^{n+1}\right\|_{0}\left\|\hat{\boldsymbol{w}}^{n+1}\right\|_{2}\left\|\hat{\boldsymbol{w}}^{n+1}-\boldsymbol{v}_{h}\right\|_{1} \\
& \leq C \Delta t\left\|\hat{\boldsymbol{e}}_{d}^{n+1}\right\|_{0}^{2}+C\left\|\hat{\boldsymbol{w}}^{n+1}-\boldsymbol{v}_{h}\right\|_{1}^{2} .
\end{aligned}
$$

The rest of the terms can also be bounded,

$$
\begin{aligned}
\left(\hat{\boldsymbol{e}}_{d}^{n+1}-\boldsymbol{e}_{d}^{n}, \hat{\boldsymbol{w}}^{n+1}-\boldsymbol{v}_{h}\right) & \leq \frac{1}{3}\left\|\hat{\boldsymbol{e}}_{d}^{n+1}-\boldsymbol{e}_{d}^{n}\right\|_{0}^{2}+C \frac{\Delta t}{h^{2}}\left\|\hat{\boldsymbol{w}}^{n+1}-\boldsymbol{v}_{h}\right\|_{0}^{2}, \\
v\left(\nabla \hat{\boldsymbol{e}}_{d}^{n+1}, \nabla\left(\hat{\boldsymbol{w}}^{n+1}-\boldsymbol{v}_{h}\right)\right) & \leq \frac{1}{8} v\left\|\hat{\boldsymbol{e}}_{d}^{n+1}\right\|_{1}^{2}+C\left\|\hat{\boldsymbol{w}}^{n+1}-\boldsymbol{v}_{h}\right\|_{1}^{2},
\end{aligned}
$$

where Assumption 7 is used for the first inequality.

Using all these inequalities in (15), invoking the stability estimates for $\hat{\boldsymbol{e}}_{d}^{n+1}$ and adding up from $n=0$ to $n=N$, we get

$$
\begin{aligned}
& \frac{1}{2} \sum_{n=0}^{N}\left(\left\|\hat{\boldsymbol{e}}_{d}^{n+1}\right\|_{0}^{2}-\left\|\boldsymbol{e}_{d}^{n}\right\|_{0}^{2}+\left\|\hat{\boldsymbol{e}}_{d}^{n+1}-\boldsymbol{e}_{d}^{n}\right\|_{0}^{2}\right)+\sum_{n=0}^{N} v \Delta t\left\|\hat{\boldsymbol{e}}_{d}^{n+1}\right\|_{1}^{2} \\
& \leq C \Delta t^{2}+\frac{6}{8} \sum_{n=0}^{N} v \Delta t\left\|\hat{\boldsymbol{e}}_{d}^{n+1}\right\|_{1}^{2}+\frac{1}{3} \sum_{n=0}^{N}\left\|\hat{\boldsymbol{e}}_{d}^{n+1}-\boldsymbol{e}_{d}^{n}\right\|_{0}^{2} \\
& \quad+C \sum_{n=0}^{N} \Delta t\left(\left\|\boldsymbol{e}_{d}^{n+1}\right\|_{0}^{2}+\left\|\boldsymbol{e}_{d}^{n+1}-\hat{\boldsymbol{e}}_{d}^{n+1}\right\|_{0}^{2}\right)+C\left(1+\sum_{n=0}^{N} v \Delta t\left\|_{d}^{n+1}\right\|_{1}^{2}\right) \tilde{\mathcal{I}}(h)^{2} \\
& \leq C\left(\tilde{\mathcal{I}}(h)^{2}+\Delta t^{2}\right)+\frac{6}{8} \sum_{n=0}^{N} v \Delta t\left\|\hat{\boldsymbol{e}}_{d}^{n+1}\right\|_{1}^{2}+\frac{1}{3} \sum_{n=0}^{N}\left\|\hat{\boldsymbol{e}}_{d}^{n+1}-\boldsymbol{e}_{d}^{n}\right\|_{0}^{2} \\
& \quad+C \sum_{n=0}^{N} \Delta t\left(\left\|\boldsymbol{e}_{d}^{n+1}\right\|_{0}^{2}+\left\|\boldsymbol{e}_{d}^{n+1}-\hat{\boldsymbol{e}}_{d}^{n+1}\right\|_{0}^{2}\right)
\end{aligned}
$$

Taking $\Delta t\left(\boldsymbol{x}_{h}-\boldsymbol{u}_{h}^{n+1}\right)$ as test function in (14b) (valid due to Assumption 3), where $\boldsymbol{x}_{h} \in \operatorname{ker} B_{h}$, we obtain

$$
\frac{1}{2}\left(\left\|\boldsymbol{e}_{d}^{n+1}\right\|_{0}^{2}-\left\|\hat{\boldsymbol{e}}_{d}^{n+1}\right\|_{0}^{2}+\left\|\boldsymbol{e}_{d}^{n+1}-\hat{\boldsymbol{e}}_{d}^{n+1}\right\|_{0}^{2}\right)=\left(\boldsymbol{e}_{d}^{n+1}-\hat{\boldsymbol{e}}_{d}^{n+1}, \boldsymbol{w}^{n+1}-\boldsymbol{x}_{h}\right) .
$$

The optimal approximation properties for functions $\boldsymbol{x}_{h} \in$ ker $B_{h}$ allow us to bound the RHS, obtaining

$$
\frac{1}{2}\left(\left\|\boldsymbol{e}_{d}^{n+1}\right\|_{0}^{2}-\left\|\hat{\boldsymbol{e}}_{d}^{n+1}\right\|_{0}^{2}+\left\|\boldsymbol{e}_{d}^{n+1}-\hat{\boldsymbol{e}}_{d}^{n+1}\right\|_{0}^{2}\right) \leq C \Delta t \mathcal{I}_{n}(h)^{2}+\frac{1}{3}\left\|\boldsymbol{e}_{d}^{n+1}-\hat{\boldsymbol{e}}_{d}^{n+1}\right\|_{0}^{2}
$$

Adding up from $n=0$ to $n=N$, we get

$$
\frac{1}{2} \sum_{n=0}^{N}\left(\left\|\boldsymbol{e}_{d}^{n+1}\right\|_{0}^{2}-\left\|\hat{\boldsymbol{e}}_{d}^{n+1}\right\|_{0}^{2}+\left\|\boldsymbol{e}_{d}^{n+1}-\hat{\boldsymbol{e}}_{d}^{n+1}\right\|_{0}^{2}\right) \leq C \tilde{\mathcal{I}}(h)^{2}+\frac{1}{3} \sum_{n=0}^{N}\left\|\boldsymbol{e}_{d}^{n+1}-\hat{\boldsymbol{e}}_{d}^{n+1}\right\|_{0}^{2}
$$


Adding (16) and (17), using the discrete Gronwall lemma (see [19]) and cancelling some terms we obtain the error estimates for the velocity. For the pressure error we take benefit from the inf-sup condition stated in Assumption 4, equation (14b) and the bounds for the velocity errors.

Theorem 1 establishes optimal convergence results for the approximated velocity when the classical first order projection method in its fully discretized form is used.

We end our analysis with a corollary that gives the convergence results obtained for the fully discrete fractional step method relying on the inf-sup condition. It is a direct consequence of Corollary 1 and Theorem 1 using the triangle inequality.

Corollary 2 (Convergence) Under the assumption of Theorem 1 and the regularity assumption $\partial_{t}^{3} \boldsymbol{f}(t) \in L^{\infty}\left(0, T ; \boldsymbol{L}^{2}(\Omega)\right)$ the following error estimates hold,

$$
\begin{aligned}
& \max _{0 \leq n \leq N}\left\{\left\|\boldsymbol{u}\left(t^{n+1}\right)-\boldsymbol{u}_{h}^{n+1}\right\|_{0}+\left\|\boldsymbol{u}\left(t^{n+1}\right)-\hat{\boldsymbol{u}}_{h}^{n+1}\right\|_{0}+\sqrt{\Delta t}\left\|\boldsymbol{u}\left(t^{n+1}\right)-\hat{\boldsymbol{u}}_{h}^{n+1}\right\|_{1}\right\} \\
& +\lambda_{n+1} \sqrt{\Delta t}\left(\sum_{n=0}^{N} \Delta t\left\|p\left(t^{n+1}\right)-p_{h}^{n+1}\right\|_{0}^{2}\right)^{1 / 2} \leq C(\tilde{\mathcal{I}}(h)+\Delta t),
\end{aligned}
$$

for $\Delta t>0$ small enough.

\section{Convergence results for a stabilized scheme using the pressure Poisson equation}

In order to obtain the pressure of the system analyzed in the previous section, a Stokeslike problem has to be solved. On the other hand, for the fractional step method studied in this section only a simple Poisson equation must be solved. This so-called Pressure Poisson equation is by far the most used approach, not only for its low computational cost, but also because of its inherent stability studied in [6,7]. At the continuous level, these two approximations are equivalent assuming some regularity properties. But at the fully discrete level, the second procedure introduces a perturbation term in the mass conservation equation.

In this section we analyze a fully discrete fractional step method using a Poisson equation for the pressure. In Sect. 4 we have obtained error estimates for the auxiliary problem at the continuous level and now we compare the fully discrete fractional step method with the auxiliary problem. We begin the analysis of the discrete problem stating the new assumptions we have had to use:

Assumption 8 There exist $\alpha_{-}>0$ and $\alpha_{+}>0$ independent of $h$ such that:

$$
\alpha_{-} h^{2} \leq \Delta t \leq \alpha_{+} h^{2}
$$

This assumption dictates the behaviour of $\Delta t$. It could seem a very restrictive hypothesis, but we will see in the following analysis that $\Delta t$ is playing the same role as the numerical stabilization parameter of stabilized finite element methods for which this assumption is mandatory (see [2]). 
Assumption 9 As in $[8,9]$, let $\nabla \mathcal{Q}_{h}$ denote the space

$$
\nabla \mathcal{Q}_{h}=\left\{\boldsymbol{v}_{h} \in \boldsymbol{L}^{2}(\Omega) \mid \boldsymbol{v}_{h}=\nabla q_{h}, q_{h} \in \mathcal{Q}_{h}\right\}
$$

and define the space $\boldsymbol{E}_{h}$ by

$$
\boldsymbol{E}_{h}=\mathcal{V}_{h}+\nabla \mathcal{Q}_{h} \subset \boldsymbol{L}^{2}(\Omega)
$$

We consider three mutually orthogonal subspaces $\boldsymbol{E}_{h, i}$ of $\boldsymbol{E}_{h}$ defined by

$$
\boldsymbol{E}_{h, 1}=\mathcal{V}_{h, 0}, \quad \boldsymbol{E}_{h, 2}=\mathcal{V}_{h, 0}^{\perp} \cap \mathcal{V}_{h}, \quad \boldsymbol{E}_{h, 3}=\mathcal{V}_{h}^{\perp} \cap \boldsymbol{E}_{h}
$$

so that

$$
\boldsymbol{E}_{h}=\boldsymbol{E}_{h, 1} \oplus \boldsymbol{E}_{h, 2} \oplus \boldsymbol{E}_{h, 3} .
$$

For $i=1,2,3$ we call $\Pi_{i}$ the $L^{2}$-projection of $\boldsymbol{E}_{h}$ onto $\boldsymbol{E}_{h, i}$, and for $i \neq j, \Pi_{i j}=$ $\Pi_{i}+\Pi_{j}$ and $\boldsymbol{E}_{h, i j}=\boldsymbol{E}_{h, i} \oplus \boldsymbol{E}_{h, j}$. We assume that there is a constant $\beta_{0}$ independent of $h$ such that

$$
\left\|\nabla q_{h}\right\|_{0} \leq \beta_{0}\left\|\Pi_{13}\left(\nabla q_{h}\right)\right\|_{0} \quad \forall q_{h} \in Q_{h},
$$

that is to say, that the second component of the decomposition of every $\nabla q_{h}$ in $\boldsymbol{E}_{h}$ can be bounded in terms of the other two. This condition can also be written in the form

$$
\inf _{q_{h} \in Q_{h}} \sup _{\boldsymbol{v}_{h} \in \boldsymbol{E}_{h, 13}} \frac{b\left(\boldsymbol{v}_{h}, q_{h}\right)}{\left\|\boldsymbol{v}_{h}\right\|_{1}\left\|q_{h}\right\|_{0}} \geq \beta_{0}>0,
$$

in a similar way to the classical inf-sup condition. However, this requirement is weaker since the space where the supremum is taken, $\boldsymbol{E}_{h, 13}$, is larger than in a classical case, $\mathcal{V}_{h, 0}=\boldsymbol{E}_{h, 1}$. Condition (18) was analized in [8], where it was shown to be satisfied by equal order simplicial finite element interpolations.

From the previous definitions we can easily see that,

$$
\Pi_{3}\left(\nabla q_{h}\right)=\Pi_{h}^{\perp}\left(\nabla q_{h}\right)
$$

for all $q_{h} \in \mathcal{Q}_{h}, \Pi_{h}(\cdot)$ being the $L^{2}$-projection onto the finite element space $\mathcal{V}_{h}$. We will also use the projector $\Pi_{\mathcal{Y}_{h, 0}}(\cdot)$, that consist of the $L^{2}$-projection onto the finite element space $\mathcal{Y}_{h, 0}$. The equations for the intermediate and end-of-step velocities are (12a) and (12c), respectively. The difference with respect to the previous section is the continuity equation, that now is replaced by a Pressure Poisson equation obtained by testing (10a) with $q_{h} \in \mathcal{Q}_{h}$ and seeking a pressure $p_{h}^{n+1} \in \mathcal{Q}_{h}$ such that

$$
\left(\nabla \cdot \hat{\boldsymbol{u}}_{h}^{n+1}, q_{h}\right)+\Delta t\left(\nabla p_{h}^{n+1}, \nabla q_{h}\right)=0 .
$$


An alternative version of the pressure Poisson equation more appropriate for the following analysis is obtained using (12b), which implies that

$$
\hat{\boldsymbol{u}}_{h}^{n+1}=\boldsymbol{u}_{h}^{n+1}+\Delta t \Pi_{\mathcal{Y}_{h, 0}}\left(\nabla p_{h}^{n+1}\right) \text {. }
$$

Using that in (20) we get

$$
\begin{aligned}
& \left(\nabla \cdot \boldsymbol{u}_{h}^{n+1}, q_{h}\right)-\Delta t\left(\Pi_{\mathcal{Y}_{h, 0}}\left(\nabla p_{h}^{n+1}\right), \nabla q_{h}\right)+\Delta t\left(\nabla p_{h}^{n+1}, \nabla q_{h}\right) \\
& \quad=\left(\nabla \cdot \boldsymbol{u}_{h}^{n+1}, q_{h}\right)+\Delta t\left(\Pi_{\mathcal{Y}_{h, 0}}^{\perp}\left(\nabla p_{h}^{n+1}\right), \nabla q_{h}\right)=0 .
\end{aligned}
$$

Then, the fully discrete method to be analyzed consists of finding $\hat{\boldsymbol{u}}_{h}^{n+1} \in \mathcal{V}_{h, 0}$, $\boldsymbol{u}_{h}^{n+1} \in \mathcal{Y}_{h, 0}$ and $p_{h}^{n+1} \in \mathcal{Q}_{h}$ such that:

$$
\begin{aligned}
\frac{1}{\delta t}\left(\hat{\boldsymbol{u}}_{h}^{n+1}-\boldsymbol{u}_{h}^{n}, \boldsymbol{v}_{h}\right)+v\left(\nabla \hat{\boldsymbol{u}}_{h}^{n+1}, \nabla \boldsymbol{v}_{h}\right)+c\left(\hat{\boldsymbol{u}}_{h}^{n+1}, \hat{\boldsymbol{u}}_{h}^{n+1}, \boldsymbol{v}_{h}\right) & =\left\langle\boldsymbol{f}\left(t^{n+1}\right), \boldsymbol{v}_{h}\right\rangle, \\
\frac{1}{\delta t}\left(\boldsymbol{u}_{h}^{n+1}-\hat{\boldsymbol{u}}_{h}^{n+1}, \boldsymbol{y}_{h}\right)+b\left(\boldsymbol{y}_{h}, p_{h}^{n+1}\right) & =0 \\
\left(\nabla \cdot \boldsymbol{u}_{h}^{n+1}, q_{h}\right)+\Delta t\left(\Pi_{\mathcal{Y}_{h, 0}}^{\perp}\left(\nabla p_{h}^{n+1}\right), \nabla q_{h}\right) & =0
\end{aligned}
$$

where we have taken the advection velocity implicitly. However, a straightforward extension of the analysis that follows allows to obtain the same estimates for the explicit case.

Subtracting (21) from the weak form of (11), it can be seen that the discrete errors satisfy the following system, which holds for all $\left(\boldsymbol{v}_{h}, \boldsymbol{y}_{h}, q_{h}\right) \in \mathcal{V}_{h, 0} \times \mathcal{Y}_{h, 0} \times \mathcal{Q}_{h}$ :

$$
\begin{aligned}
\frac{1}{\delta t}\left(\hat{\boldsymbol{e}}_{d}^{n+1}-\boldsymbol{e}_{d}^{n}, \boldsymbol{v}_{h}\right)+v\left(\nabla \hat{\boldsymbol{e}}_{d}^{n+1}, \nabla \boldsymbol{v}_{h}\right) & =c\left(\hat{\boldsymbol{u}}_{h}^{n+1}, \hat{\boldsymbol{u}}_{h}^{n+1}, \boldsymbol{v}_{h}\right) \\
& -c\left(\boldsymbol{u}\left(t^{n+1}\right), \boldsymbol{u}\left(t^{n+1}\right), \boldsymbol{v}_{h}\right), \\
\frac{1}{\delta t}\left(\boldsymbol{e}_{d}^{n+1}-\hat{\boldsymbol{e}}_{d}^{n+1}, \boldsymbol{y}_{h}\right)+\left(\nabla r_{d}^{n+1}, \boldsymbol{y}_{h}\right) & =0 \\
\left(\nabla \cdot \boldsymbol{e}_{d}^{n+1}, q_{h}\right)-\Delta t\left(\Pi \stackrel{\mathcal{Y}}{h, 0}^{\perp}\left(\nabla p_{h}^{n+1}\right), \nabla q_{h}\right) & =0
\end{aligned}
$$

where we have denoted the error sequences as in the previous section. We also use the following notation for the interpolation error:

$$
\begin{aligned}
\tilde{\mathcal{I}}_{n}(h)= & \frac{1}{h} \inf _{\boldsymbol{v}_{h} \in \mathcal{V}_{h, 0}}\left\|\hat{\boldsymbol{w}}^{n+1}-\boldsymbol{v}_{h}\right\|_{0}+\frac{1}{h} \inf _{\boldsymbol{y}_{h} \in \mathcal{Y}_{h, 0}}\left\|\boldsymbol{w}^{n+1}-\boldsymbol{y}_{h}\right\|_{0}+\inf _{\boldsymbol{v}_{h} \in \mathcal{V}_{h, 0}}\left\|\hat{\boldsymbol{w}}^{n+1}-\boldsymbol{v}_{h}\right\|_{1} \\
& +\inf _{q_{h} \in \mathcal{Q}_{h}}\left\|\phi^{n+1}-q_{h}\right\|_{0}+h \inf _{q_{h} \in Q_{h}}\left\|\phi^{n+1}-q_{h}\right\|_{1}+h \inf _{\boldsymbol{y}_{h} \in \mathcal{Y}_{h, 0}}\left\|\nabla \phi^{n+1}-\boldsymbol{y}_{h}\right\|_{0} \\
\tilde{\mathcal{I}}(h)= & \max _{0 \leq n \leq N} \tilde{\mathcal{I}}_{n}(h) .
\end{aligned}
$$

The last term of $\tilde{\mathcal{I}}_{n}(h)$ differs from the expression used in the previous analysis (under the inf-sup condition). This term is of special interest, as it will be commented below. 
Again, the fact that this interpolation function depends on the auxiliary unknowns does not affect the convergence resutls, due to Assumption 8 and Theorem 1 .

Before proving the error estimates, we need a previous lemma that gives us a bound for the pressure error. The lemma reads as follows:

Lemma 3 Under Assumption 9, the following bound holds in the range $0 \leq n \leq N$ :

$$
\left\|\nabla r_{d}^{n+1}\right\|_{0} \leq C\left(\frac{1}{h} \tilde{\mathcal{I}}_{n}(h)+\frac{1}{\delta t}\left\|\boldsymbol{e}_{d}^{n+1}-\hat{\boldsymbol{e}}_{d}^{n+1}\right\|_{0}+\left\|\Pi_{3}\left(\nabla p_{h}^{n+1}\right)\right\|_{0}\right)
$$

for all $\Delta t>0$.

Proof By the triangle inequality and previous definitions, we have

$$
\begin{aligned}
\left\|\nabla r_{d}^{n+1}\right\|_{0}= & \left\|\nabla\left(\phi^{n+1}-p_{h}^{n+1}\right)\right\|_{0} \\
\leq & \left\|\nabla \phi^{n+1}-\Pi_{12}\left(\nabla q_{h}\right)\right\|_{0}+\left\|\Pi_{1}\left(\nabla q_{h}-\nabla p_{h}^{n+1}\right)\right\|_{0} \\
& +\left\|\Pi_{2}\left(\nabla q_{h}-\nabla p_{h}^{n+1}\right)\right\|_{0}+\left\|\Pi_{3}\left(\nabla p_{h}^{n+1}\right)\right\|_{0} \\
= & \widetilde{\mathrm{I}}+\widetilde{\mathrm{II}}+\widetilde{\mathrm{III}}+\widetilde{\mathrm{IV}} .
\end{aligned}
$$

We bound these terms separately. For the first term we can easily obtain,

$$
\begin{aligned}
\widetilde{\mathrm{I}} & \leq\left\|\Pi_{3}\left(\nabla \phi^{n+1}\right)\right\|_{0}+\left\|\Pi_{12}\left(\nabla \phi^{n+1}-\nabla q_{h}\right)\right\|_{0} \\
& \leq\left\|\Pi_{3}\left(\nabla \phi^{n+1}-\boldsymbol{y}_{h}\right)\right\|_{0}+\left\|\phi^{n+1}-q_{h}\right\|_{1} \\
& \leq\left\|\nabla \phi^{n+1}-\boldsymbol{y}_{h}\right\|_{0}+\left\|\phi^{n+1}-q_{h}\right\|_{1},
\end{aligned}
$$

for all $\boldsymbol{y}_{h} \in \mathcal{Y}_{h, 0} \subset \boldsymbol{E}_{h, 12}$ For the second term we have,

$$
\begin{aligned}
(\widetilde{\mathrm{II}})^{2} & =\left\|\Pi_{1}\left(\nabla q_{h}-\nabla p_{h}^{n+1}\right)\right\|_{0}^{2}=\left(\nabla q_{h}-\nabla p_{h}^{n+1}, \Pi_{1}\left(\nabla q_{h}-\nabla p_{h}^{n+1}\right)\right) \\
& =\left(\nabla q_{h}-\nabla \phi^{n+1}, \Pi_{1}\left(\nabla q_{h}-\nabla p_{h}^{n+1}\right)\right)+\left(\nabla r_{d}^{n+1}, \Pi_{1}\left(\nabla q_{h}-\nabla p_{h}^{n+1}\right)\right) \\
& =\widetilde{\mathrm{I}}_{a}+\widetilde{\mathrm{II}}_{b} .
\end{aligned}
$$

The first term is easily bounded,

$$
\widetilde{\mathrm{II}}_{a} \leq\left\|\phi^{n+1}-q_{h}\right\|_{1}\left\|\Pi_{1}\left(\nabla q_{h}-\nabla p_{h}^{n+1}\right)\right\|_{0} .
$$

For the second term, we take $\left.\boldsymbol{y}_{h}=\Pi_{1}\left(\nabla q_{h}-\nabla p_{h}^{n+1}\right)\right)$ in (22b), obtaining

$$
\begin{aligned}
\widetilde{\mathrm{I}}_{b} & =\left(\nabla r_{d}^{n+1}, \Pi_{1}\left(\nabla q_{h}-\nabla p_{h}^{n+1}\right)\right) \\
& =-\frac{1}{\delta t}\left(\boldsymbol{e}_{d}^{n+1}-\hat{\boldsymbol{e}}_{d}^{n+1}, \Pi_{1}\left(\nabla q_{h}-\nabla p_{h}^{n+1}\right)\right) \\
& \leq \frac{1}{\delta t}\left\|\boldsymbol{e}_{d}^{n+1}-\hat{\boldsymbol{e}}_{d}^{n+1}\right\|_{0}\left\|\Pi_{1}\left(\nabla q_{h}-\nabla p_{h}^{n+1}\right)\right\|_{0} .
\end{aligned}
$$


Then,

$$
\widetilde{\mathrm{II}} \leq\left\|\phi^{n+1}-q_{h}\right\|_{1}+\frac{1}{\delta t}\left\|\boldsymbol{e}_{d}^{n+1}-\hat{\boldsymbol{e}}_{d}^{n+1}\right\|_{0}
$$

Moreover, due to Assumption 9 we can obtain

$$
\begin{aligned}
\widetilde{\mathrm{III}} & \leq C\left(\left\|\Pi_{1}\left(\nabla q_{h}-\nabla p_{h}^{n+1}\right)\right\|_{0}+\left\|\Pi_{3}\left(\nabla q_{h}-\nabla p_{h}^{n+1}\right)\right\|_{0}\right) \\
& \leq C\left(\widetilde{\mathrm{II}}+\left\|\Pi_{3}\left(\nabla q_{h}\right)\right\|_{0}+\left\|\Pi_{3}\left(\nabla p_{h}^{n+1}\right)\right\|_{0}\right) \\
& \left.\leq C \widetilde{\mathrm{I}}+\widetilde{\mathrm{II}}+\left\|\nabla \phi^{n+1}-\nabla q_{h}\right\|_{0}+2\left\|\Pi_{3}\left(\nabla p_{h}^{n+1}\right)\right\|_{0}\right) .
\end{aligned}
$$

Then, taking the infimum with respect to $q_{h} \in Q_{h}$, we prove the statement of the theorem.

The error estimates we want to obtain are established in the next theorem:

Theorem 2 Under Assumptions 1, 2, 8, 6 and 9, we have

$$
\begin{aligned}
\max _{0 \leq n \leq N}\{ & \left.\left\|\boldsymbol{e}_{d}^{N+1}\right\|_{0}^{2}+\left\|\hat{\boldsymbol{e}}_{d}^{N+1}\right\|_{0}^{2}\right\}+\sum_{n=0}^{N} v \Delta t\left\{\left\|\hat{\boldsymbol{e}}_{d}^{n+1}\right\|_{1}^{2}+\left\|\boldsymbol{e}_{d}^{n+1}\right\|_{1}^{2}\right\} \\
& +\sum_{n=0}^{N}\left\{\left\|\boldsymbol{e}_{d}^{n+1}-\hat{\boldsymbol{e}}_{d}^{n+1}\right\|_{1}^{2}+\left\|\hat{\boldsymbol{e}}_{d}^{n+1}-\boldsymbol{e}_{d}^{n+1}\right\|_{1}^{2}\right\} \\
& +\sum_{n=0}^{N} \Delta t^{2}\left\|\nabla r_{d}^{n+1}\right\|_{0}^{2} \leq C\left(\tilde{\mathcal{I}}(h)^{2}+\Delta t^{2}\right)
\end{aligned}
$$

for $\Delta t>0$ small enough.

Proof The first step of this proof is similar to that in Theorem 1. We take $\Delta t\left(\boldsymbol{v}_{h}-\hat{\boldsymbol{u}}_{h}^{n+1}\right)$ as a test function in (22a). Using some bounds from Theorem 1, invoking the stability estimates for $\hat{\boldsymbol{e}}_{d}^{n+1}$ and adding up from $n=0$ to $n=N$, we get

$$
\begin{aligned}
& \frac{1}{2} \sum_{n=0}^{N}\left(\left\|\hat{\boldsymbol{e}}_{d}^{n+1}\right\|_{0}^{2}-\left\|\boldsymbol{e}_{d}^{n}\right\|_{0}^{2}+\left\|\hat{\boldsymbol{e}}_{d}^{n+1}-\boldsymbol{e}_{d}^{n}\right\|_{0}^{2}\right)+\sum_{n=0}^{N} v \Delta t\left\|\hat{\boldsymbol{e}}_{d}^{n+1}\right\|_{1}^{2} \\
& \leq C\left(\tilde{\mathcal{I}}(h)^{2}+\Delta t^{2}\right)+\frac{6}{8} \sum_{n=0}^{N} v \Delta t\left\|\hat{\boldsymbol{e}}_{d}^{n+1}\right\|_{1}^{2}+\frac{1}{3} \sum_{n=0}^{N}\left\|\hat{\boldsymbol{e}}_{d}^{n+1}-\boldsymbol{e}_{d}^{n}\right\|_{0}^{2} \\
& \quad+C \sum_{n=0}^{N} \Delta t\left(\left\|\boldsymbol{e}_{d}^{n+1}\right\|_{0}^{2}+\left\|\boldsymbol{e}_{d}^{n+1}-\hat{\boldsymbol{e}}_{d}^{n+1}\right\|_{0}^{2}\right)
\end{aligned}
$$


The proof goes on taking $\Delta t\left(\boldsymbol{v}_{h}-\boldsymbol{u}_{h}^{n+1}\right)$ as a test function in (22b), getting

$$
\begin{aligned}
& \frac{1}{2}\left(\left\|\boldsymbol{e}_{d}^{n+1}\right\|_{0}^{2}-\left\|\hat{\boldsymbol{e}}_{d}^{n+1}\right\|_{0}^{2}+\left\|\boldsymbol{e}_{d}^{n+1}-\hat{\boldsymbol{e}}_{d}^{n+1}\right\|_{0}^{2}\right)+\Delta t\left(\nabla r_{d}^{n+1}, \boldsymbol{e}_{d}^{n+1}\right) \\
& \quad=\left(\boldsymbol{e}_{d}^{n+1}-\hat{\boldsymbol{e}}_{d}^{n+1}, \boldsymbol{w}^{n+1}-\boldsymbol{v}_{h}\right)+\Delta t\left(\nabla r_{d}^{n+1}, \boldsymbol{w}^{n+1}-\boldsymbol{v}_{h}\right)
\end{aligned}
$$

We bound the first term as follows,

$$
\left(\boldsymbol{e}_{d}^{n+1}-\hat{\boldsymbol{e}}_{d}^{n+1}, \boldsymbol{w}^{n+1}-\boldsymbol{v}_{h}\right) \leq \frac{1}{6}\left\|\boldsymbol{e}_{d}^{n+1}-\hat{\boldsymbol{e}}_{d}^{n+1}\right\|_{0}^{2}+C \frac{\Delta t}{h^{2}}\left\|\boldsymbol{w}^{n+1}-\boldsymbol{v}_{h}\right\|_{0}^{2}
$$

Using Lemma 3 we can bound the second term in the RHS of (24),

$$
\begin{aligned}
& \left(\nabla r_{d}^{n+1}, \boldsymbol{w}^{n+1}-\boldsymbol{v}_{h}\right) \leq C\left\|\nabla r_{d}^{n+1}\right\|_{0}\left\|\boldsymbol{w}^{n+1}-\boldsymbol{v}_{h}\right\|_{0} \\
& \quad \leq C\left(\tilde{\mathcal{I}}_{n}(h)+\frac{1}{\delta t}\left\|\boldsymbol{e}_{d}^{n+1}-\hat{\boldsymbol{e}}_{d}^{n+1}\right\|_{0}+\left\|\Pi_{3}\left(\nabla p_{h}^{n+1}\right)\right\|_{0}\right)\left\|\boldsymbol{w}^{n+1}-\boldsymbol{v}_{h}\right\|_{0} \\
& \quad \leq C \tilde{\mathcal{I}}_{n}(h)^{2}+\frac{1}{6 \Delta t}\left\|\boldsymbol{e}_{d}^{n+1}-\hat{\boldsymbol{e}}_{d}^{n+1}\right\|_{0}^{2}+\frac{\Delta t}{3}\left\|\Pi_{3}\left(\nabla p_{h}^{n+1}\right)\right\|_{0}^{2} .
\end{aligned}
$$

Adding up from $n=0$ to $n=N$, we get

$$
\begin{aligned}
& \frac{1}{2} \sum_{n=0}^{N}\left(\left\|\boldsymbol{e}_{d}^{n+1}\right\|_{0}^{2}-\left\|\hat{\boldsymbol{e}}_{d}^{n+1}\right\|_{0}^{2}+\left\|\boldsymbol{e}_{d}^{n+1}-\hat{\boldsymbol{e}}_{d}^{n+1}\right\|_{0}^{2}\right)+\sum_{n=0}^{N} \Delta t\left(\nabla r_{d}^{n+1}, \boldsymbol{e}_{d}^{n+1}\right) \\
& \quad \leq \frac{1}{3} \sum_{n=0}^{N}\left\|\boldsymbol{e}_{d}^{n+1}-\hat{\boldsymbol{e}}_{d}^{n+1}\right\|_{0}^{2}+C \tilde{\mathcal{I}}(h)^{2}+\frac{1}{3} \sum_{n=0}^{N} \Delta t^{2}\left\|\Pi_{3}\left(\nabla p_{h}^{n+1}\right)\right\|_{0}^{2}
\end{aligned}
$$

Now we take $\Delta t\left(q_{h}-p_{h}^{n+1}\right)$ as a test function in (22c), obtaining

$$
\begin{aligned}
& \Delta t\left(\nabla \cdot \boldsymbol{e}_{d}^{n+1}, r_{d}^{n+1}\right)+\Delta t^{2}\left\|\Pi_{\mathcal{Y}_{h, 0}}^{\perp}\left(\nabla p_{h}^{n+1}\right)\right\|_{0}^{2} \\
& =\Delta t\left(\nabla \cdot \boldsymbol{e}_{d}^{n+1}, \phi^{n+1}-q_{h}\right)+\Delta t^{2}\left(\Pi_{\mathcal{Y}_{h, 0}}^{\perp}\left(\nabla p_{h}^{n+1}\right), \nabla q_{h}\right) \\
& =\Delta t\left(\nabla \cdot \boldsymbol{e}_{d}^{n+1}, \phi^{n+1}-q_{h}\right)-\Delta t^{2}\left(\Pi_{\mathcal{Y}_{h, 0}}^{\perp}\left(\nabla p_{h}^{n+1}\right), \boldsymbol{y}_{h}-\nabla q_{h}\right)
\end{aligned}
$$

for any function $\boldsymbol{y}_{h} \in \mathcal{Y}_{h, 0}$. We bound the first term of the RHS invoking Assumption 8,

$$
\begin{aligned}
\left(\nabla \cdot \boldsymbol{e}_{d}^{n+1}, \phi^{n+1}-q_{h}\right)= & -\left(\boldsymbol{e}_{d}^{n+1}-\hat{\boldsymbol{e}}_{d}^{n+1}, \nabla \phi^{n+1}-\nabla q_{h}\right)+\left(\nabla \cdot \hat{\boldsymbol{e}}_{d}^{n+1}, \phi^{n+1}-q_{h}\right) \\
\leq & \frac{1}{8} v\left\|\hat{\boldsymbol{e}}_{d}^{n+1}\right\|_{1}^{2}+C\left\|\phi^{n+1}-q_{h}\right\|_{0}^{2}+\frac{1}{6 \Delta t}\left\|\boldsymbol{e}_{d}^{n+1}-\hat{\boldsymbol{e}}_{d}^{n+1}\right\|_{0}^{2} \\
& +C h^{2}\left\|\phi^{n+1}-q_{h}\right\|_{1}^{2},
\end{aligned}
$$


and using the triangular inequality and Young's inequality, we have,

$$
\begin{aligned}
& \left.-\left(\Pi \frac{\mathcal{Y}}{h, 0}^{\perp}\left(\nabla p_{h}^{n+1}\right), \boldsymbol{y}_{h}-\nabla q_{h}\right)\right) \leq\left\|\Pi \frac{\mathcal{Y}}{h, 0}^{\perp}\left(\nabla p_{h}^{n+1}\right)\right\|_{0}\left\|\boldsymbol{y}_{h}-\nabla q_{h}\right\|_{0} \\
& \leq \frac{1}{3}\left\|\Pi \frac{\mathcal{Y}}{h, 0}^{\perp}\left(\nabla p_{h}^{n+1}\right)\right\|_{0}^{2}+C \frac{h^{2}}{\Delta t}\left\|\nabla \phi^{n+1}-\boldsymbol{y}_{h}\right\|_{0}^{2}+C \frac{h^{2}}{\Delta t}\left\|\phi^{n+1}-q_{h}\right\|_{1}^{2} .
\end{aligned}
$$

Adding up from $n=0$ to $n=N$, we have

$$
\begin{aligned}
& \sum_{n=0}^{N} \Delta t\left(\nabla \cdot \boldsymbol{e}_{d}^{n+1}, r_{d}^{n+1}\right)+\sum_{n=0}^{N} \Delta t^{2}\left\|\Pi \frac{\mathcal{Y}}{h, 0}_{h}\left(\nabla p_{h}^{n+1}\right)\right\|_{0}^{2} \\
& \leq \frac{1}{8} \sum_{n=0}^{N} \nu \Delta t\left\|\hat{\boldsymbol{e}}_{d}^{n+1}\right\|_{1}^{2}+\frac{1}{6} \sum_{n=0}^{N}\left\|\boldsymbol{e}_{d}^{n+1}-\hat{\boldsymbol{e}}_{d}^{n+1}\right\|_{0}^{2} \\
& \quad+\sum_{n=0}^{N} \frac{\Delta t^{2}}{3}\left\|\Pi_{\mathcal{Y}_{h, 0}}^{\perp}\left(\nabla p_{h}^{n+1}\right)\right\|_{0}^{2}+C \tilde{\mathcal{I}}(h)^{2} .
\end{aligned}
$$

Taking into account the fact that $\mathcal{Y}_{h, 0} \subset \mathcal{V}_{h}$, we can easily infer that, for any function $q_{h} \in \mathcal{Q}_{h}$,

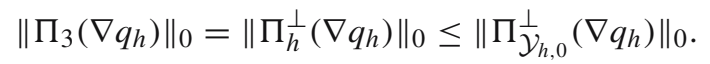

Summing up (23), (25) and (27), using (28) and applying Gronwall's lemma we finally get

$$
\begin{aligned}
\left\|\boldsymbol{e}_{d}^{N+1}\right\|_{0}^{2} & +\sum_{n=0}^{N}\left(\left\|\hat{\boldsymbol{e}}_{d}^{n+1}-\boldsymbol{e}_{d}^{n}\right\|_{0}^{2}+\left\|\boldsymbol{e}_{d}^{n+1}-\hat{\boldsymbol{e}}_{d}^{n+1}\right\|_{0}^{2}\right)+\sum_{n=0}^{N} v \Delta t\left\|\hat{\boldsymbol{e}}_{d}^{n+1}\right\|_{1}^{2} \\
& +\sum_{n=0}^{N} \Delta t^{2}\left\|\Pi \frac{\mathcal{Y}}{h, 0}^{\perp}\left(\nabla p_{h}^{n+1}\right)\right\|_{0}^{2} \leq C\left(\tilde{\mathcal{I}}(h)^{2}+\Delta t^{2}\right) .
\end{aligned}
$$

From the first and third term of the left hand side of (29) we get the bound for $\left\|\hat{\boldsymbol{e}}_{d}^{N+1}\right\|_{0}$. The bound for the pressure error is straightforward from Lemma 3, (28) and (29). This completes the proof of the theorem.

The previous a priori error estimate is optimal.

Remark 3 The new term of the interpolation error function,

$$
h \inf _{\boldsymbol{y}_{h} \in \mathcal{Y}_{h, 0}}\left\|\nabla \phi^{n+1}-\boldsymbol{y}_{h}\right\|_{0}
$$

keeps the optimality of the error bound. Nevertheless, from the previous theorem we can infer that, if we decide to seek the end-of-step velocity $\boldsymbol{u}_{h}^{n+1}$ in the finite element space $\mathcal{V}_{h, 0}$ (that is, to impose Dirichlet boundary conditions over $\boldsymbol{u}_{h}^{n+1}$ ), the situation 
gets worse. This is the case of pressure segregation methods obtained at the discrete level. The error estimates for a first order fractional step method obtained at the dicrete level is optimal (under Assumption 8) only for linear elements. The problem arises from the fact that now the new interpolation term is:

$$
h \inf _{\boldsymbol{v}_{h, 0} \in \mathcal{V}_{h, 0}}\left\|\nabla \phi^{n+1}-\boldsymbol{v}_{h}\right\|_{0} .
$$

That is the approximation of a function in $\boldsymbol{L}^{2}(\Omega)$ that does not have to vanish on the boundary (in fact, its trace is not even defined, but only its normal component) by finite element functions $\boldsymbol{v}_{h} \in \mathcal{V}_{h, 0}$ with zero trace. Let us consider a finite element function $\boldsymbol{x}_{h} \in \mathcal{V}_{h}$, and the unique decomposition:

$$
\boldsymbol{x}_{h}=\boldsymbol{x}_{h, 0}+\boldsymbol{x}_{h, \Gamma}
$$

where $\boldsymbol{x}_{h, 0} \in \mathcal{V}_{h, 0}$ and $\boldsymbol{x}_{h, \Gamma}$ is a finite element function that vanishes at the inner nodes. From basic properties of finite element shape functions and local inverse inequalities (see [12]) we can easily get:

$$
\left\|\boldsymbol{x}_{h, \Gamma}\right\|_{0} \leq C h^{1 / 2}\left\|\boldsymbol{x}_{h}\right\|_{\boldsymbol{L}^{2}(\Gamma)} .
$$

Then, we can easily bound the error term under consideration as:

$$
h \inf _{\boldsymbol{v}_{h} \in \mathcal{V}_{h, 0}}\left\|\nabla \phi^{n+1}-\boldsymbol{v}_{h}\right\|_{0} \leq h \inf _{\boldsymbol{v}_{h} \in \mathcal{V}_{h}}\left\{\left\|\nabla \phi^{n+1}-\boldsymbol{v}_{h}\right\|_{0}+C h^{1 / 2}\left\|\boldsymbol{v}_{h}\right\|_{L^{2}(\Gamma)}\right\} .
$$

This result does not allow to improve a $h^{3 / 2}$ order of accuracy in space and we are not aware of any improved error estimate for this expression. This misbehavior would be particularly relevant for high order incremental projection methods.

We end our analysis with a corollary that gives the convergence results obtained for the fully discrete fractional step method using a Pressure Poisson equation.

Corollary 3 (Convergence) Under the assumptions of Theorem 2 and the regularity assumption $\partial_{t}^{3} \boldsymbol{f}(t) \in L^{\infty}\left(0, T ; \boldsymbol{L}^{2}(\Omega)\right)$, the following error estimates hold:

$$
\begin{gathered}
\max _{0 \leq n \leq N}\left\{\left\|\boldsymbol{u}\left(t^{n+1}\right)-\boldsymbol{u}_{h}^{n+1}\right\|_{0}+\left\|\boldsymbol{u}\left(t^{n+1}\right)-\hat{\boldsymbol{u}}_{h}^{n+1}\right\|_{0}+\sqrt{\Delta t}\left\|\boldsymbol{u}\left(t^{n+1}\right)-\hat{\boldsymbol{u}}_{h}^{n+1}\right\|_{1}\right\} \\
+\lambda_{n+1} \sqrt{\Delta t}\left(\sum_{n=0}^{N} \Delta t\left\|p\left(t^{n+1}\right)-p_{h}^{n+1}\right\|_{0}^{2}\right) \leq C(\tilde{\mathcal{I}}(h)+\Delta t),
\end{gathered}
$$

for $\Delta t>0$ small enough. 


\section{Conclusions}

We have obtained optimal convergence and stability results for the fully discrete version of the classical first order projection method. The strategy pursued is based on the introduction of an auxiliary problem that has allowed the analysis of the final discrete problem. Using this strategy not only optimal error estimates have been obtained under the inf-sup condition, but also using a pressure Poisson equation (exploiting the inherent pressure of the system that we get by introducing this equation). As far as we know this is the first attempt for the obtention of convergence results for the fully discrete projection method and also the first one without the inf-sup assumption.

Moreover we have infered from the numerical analysis that projection methods obtained by splitting the discrete system, where Dirichlet boundary conditions are applied over the the end-of-step velocity, show optimal convergence only for linear elements.

\section{References}

1. Badia, S.: Stabilized Pressure Segregation Methods and their Application to Fluid-Structure Interaction Problems. PhD Thesis, Escola Tècnica Superior d'Enginyers de Camins, Canals i Ports, Universitat Politècnica de Catalunya, Barcelona (2006)

2. Blasco, J., Codina, R.: Space and time error estimates for a first order, pressure stabilized finite element method for the incompressible Navier-Stokes equations. Appl. Numer. Math. 38, 475-497 (2001)

3. Brenner, S.C., Scott, L.R.: The Mathematical Theory of Finite Element Methods. Springer, Heidelberg (1994)

4. Chorin, A.J.: The Numerical Solution of the Navier-Stokes Equations for an Incompressible Fluid. AEC Research and Development Report, NYO-1480-82. New York University, New York (1967)

5. Chorin, A.J.: Numerical solution of the Navier-Stokes equations. Math. Comput. 22, 745-762 (1968)

6. Codina, R.: Pressure stability in fractional step finite element methods for incompressible flows. J. Comput. Phys. 170, 112-140 (2001)

7. Codina, R., Badia, S.: On some pressure segregation methods of fractional-step type for the finite element approximation of incompressible flow problems. Comput. Meth. Appl. Mech. Eng. 195, 29002918 (2006)

8. Codina, R., Blasco, J.: A finite element formulation for the Stokes problem allowing equal velocitypressure interpolation. Comput. Meth. Appl. Mech. Eng. 143, 373-391 (1997)

9. Codina, R., Blasco, J.: Analysis of a pressure-stabilized finite element approximation of the stationary Navier-Stokes equations. Numer. Math. 87, 59-81 (2000)

10. Constantin, P., Foias, C.: Navier-Stokes Equations. University of Chicago Press, Chicago and London (1988)

11. de Rham, G.: Variétés Différentiables Formes, Courants, Formes Harmoniques. Hermann, Paris (1973)

12. Fernández, M.A., Gerbeau, J.F., Grandmont, C.: A projection semi-implicit scheme for the coupling of an elastic structure with an incompressible fluid. Int. J. Numer. Methods Eng. 69(4), 794-821 (2007)

13. Girault, V., Raviart, P.A.: Finite Element Methods for Navier-Stokes Equations. Springer, Heidelberg (1986)

14. Gresho, P.M.: On the theory of semi-implicit projection methods for viscous incompressible flow and its implementation via a finite element method that also introduces a nearly consistent mass matrix. Part I: Theory. Int. J. Numer. Methods Fluids 11, 587-620 (1990)

15. Guermond, J.L.: Remarques sur les méthodes de projection pour l'approximation des équations de Navier-Stokes. Numer. Math. 67, 465-473 (1994)

16. Guermond, J.L., Quartapelle, L.: On stability and convergence of projection methods based on pressure Poisson equation. Int. J. Numer. Methods Fluids 26, 1039-1053 (1998)

17. Guermond, J.L., Quartapelle, L.: On the approximation of the unsteady Navier-Stokes equations by finite element projection methods. Numer. Math. 80, 207-238 (1998) 
18. Heywood, J.G., Rannacher, R.: Finite element approximation of the nonstationary Navier-Stokes problem. I: Regularity of solutions and second-order error estimates for spatial disetization. SIAM J. Numer. Anal. 19, 275-311 (1982)

19. Heywood, J.G., Rannacher, R.: Finite element approximation of the nonstationary Navier-Stokes problem. IV: Error analysis for second-order time disetization. SIAM J. Numer. Anal. 27, 353384 (1990)

20. Ladyzhenskaya, O.: The Mathematical Theory of Viscous Incompressible Flow. Gordon and Breach, New York (1969)

21. Perot, J.B.: An analysis of the fractional step method. J. Comput. Phys. 108, 51-58 (1993)

22. Prohl, A.: Projection and Quasi-Compressibility Methods for Solving the Incompressible NavierStokes Equations. B.G. Teubner Stuttgart (1997)

23. Rannacher, R.: On Chorin's Projection Method for Incompressible Navier-Stokes Equations, Lecture Notes in Mathematics, vol. 1530, pp. 167-183. Springer, Berlin (1992)

24. Shen, J.: On error estimates for some higher order projection and penalty-projection methods for Navier-Stokes equations. Numer. Math. 62, 49-73 (1992)

25. Shen, J.: Remarks on the pressure error estimates for the projection methods. Numer. Math. 67, 513520 (1994)

26. Temam, R.: Sur l'approximation de la solution des équations de Navier-Stokes par la méthode des pas fractionaires (I). Arch. Ration. Mech. Anal. 32, 135-153 (1969)

27. Temam, R.: Sur l'approximation de la solution des équations de Navier-Stokes par la méthode des pas fractionaires (II). Arch. Ration. Mech. Anal. 33, 377-385 (1969)

28. Temam, R.: Navier-Stokes Equations. North-Holland, Amsterdam (1984)

29. Temam, R.: Remark on the pressure boundary condition for the projection method. Theor. Comput. Fluid Dyn. 3, 181-184 (1991) 